\title{
ON THE EVALUATION OF SINGULAR INVARIANTS FOR CANONICAL GENERATORS OF CERTAIN GENUS ONE ARITHMETIC GROUPS
}

\author{
JAY JORGENSON, LEJLA SMAJLOVIĆ, AND HOLGER THEN
}

\begin{abstract}
Let $N$ be a positive square-free integer such that the discrete group $\Gamma_{0}(N)^{+}$has genus one. In a previous article, we constructed canonical generators $x_{N}$ and $y_{N}$ of the holomorphic function field associated to $\Gamma_{0}(N)^{+}$as well as an algebraic equation $P_{N}\left(x_{N}, y_{N}\right)=0$ with integer coefficients satisfied by these generators. In the present paper, we study the singular moduli problem corresponding to $x_{N}$ and $y_{N}$, by which we mean the arithmetic nature of the numbers $x_{N}(\tau)$ and $y_{N}(\tau)$ for any CM point $\tau$ in the upper half plane $\mathbb{H}$. If $\tau$ is any CM point which is not equivalent to an elliptic point of $\Gamma_{0}(N)^{+}$, we prove that the complex numbers $x_{N}(\tau)$ and $y_{N}(\tau)$ are algebraic integers. Going further, we characterize the algebraic nature of $x_{N}(\tau)$ as the generator of a certain ring class field of $\mathbb{Q}(\tau)$ of prescribed order and discriminant depending on properties of $\tau$ and level $N$. The theoretical considerations are supplemented by computational examples. As a result, several explicit evaluations are given for various $N$ and $\tau$, and further arithmetic consequences of our analysis are presented. In one example, we explicitly construct a set of minimal polynomials for the Hilbert class field of $\mathbb{Q}(\sqrt{-74})$ whose coefficients are less than $2.2 \times 10^{4}$, whereas the minimal polynomials obtained from the Hauptmodul of $\operatorname{PSL}(2, \mathbb{Z})$ has coefficients as large as $6.6 \times 10^{73}$.
\end{abstract}

\section{INTRODUCTION}

For any square-free integer $N \geq 1$, the subset of $\operatorname{SL}(2, \mathbb{R})$ defined by

$$
\Gamma_{0}(N)^{+}=\left\{e^{-1 / 2}\left(\begin{array}{ll}
a & b \\
c & d
\end{array}\right) \in \mathrm{SL}(2, \mathbb{R}): \quad a d-b c=e, \quad a, b, c, d, e \in \mathbb{Z}, \quad e|N, e| a, e|d, N| c\right\}
$$

is an arithmetic subgroup of $\operatorname{SL}(2, \mathbb{R})$. We denote by $\overline{\Gamma_{0}(N)^{+}}=\Gamma_{0}(N)^{+} /\{ \pm \mathrm{Id}\}$ the corresponding subgroup of $\operatorname{PSL}(2, \mathbb{R})$ and by $Y_{N}^{+}=\overline{\Gamma_{0}(N)^{+}} \backslash \mathbb{H}$ the (compactified) surface obtained by the action of $\overline{\Gamma_{0}(N)^{+}}$on the upper half plane $\mathbb{H}$. As shown in [7, there are precisely 38 squarefree numbers $N$ such that the surface $Y_{N}^{+}$has genus one, in which case we will also say that groups $\Gamma_{0}(N)^{+}$have genus one; these groups will be the focus of study in the present paper. For arbitrary square-free $N$, the quotient space $Y_{N}^{+}$, identified with the fundamental domain of the action of $\overline{\Gamma_{0}(N)^{+}}$on the upper half plane $\mathbb{H}$, has one cusp which we denote by $i \infty$.

In [8], we proved that the function field of meromorphic functions associated to $\Gamma_{0}(N)^{+}$of genus one admits two generators $x_{N}$ and $y_{N}$ whose $q$-expansions have integer coefficients after the lead coefficient has been normalized to equal one. The poles of $x_{N}$ and $y_{N}$ at the cusp $i \infty$ have order two and three, respectively, and each function is further normalized by setting the constant term in its $q$-expansion to be zero. Additionally, $x_{N}$ and $y_{N}$ satisfy an equation of the form

$$
y_{N}^{2}=x_{N}^{3}+A_{N} x_{N} y_{N}+B_{N} x_{N}^{2}+C_{N} y_{N}+D_{N} x_{N}+E_{N},
$$

where the integers $A_{N}, B_{N}, C_{N}, D_{N}$ and $E_{N}$ are explicitly computed and are given in [8].

In this paper we begin the study of the arithmetic nature of the values of $x_{N}(\tau)$ and $y_{N}(\tau)$ for points $\tau$ in the upper half plane $\mathbb{H}$ which are complex multiplication (CM) points, meaning that $a \tau^{2}+b \tau+c=0$ for integers $a, b$, and $c$, possibly with certain conditions depending on $N$. The questions we study are motivated by known results in the case of genus zero groups of the form $\Gamma_{0}(N)^{+}$, which we now briefly recall.

If $\Gamma_{0}(N)^{+}$has genus zero, then the associated function field has one generator, denoted by $j_{N}$. We assume $j_{N}$ is normalized by its $q$-expansion at $i \infty$ to have a first order pole, residue equal to one, constant term equal to zero, and holomorphic elsewhere on $\mathbb{H}$. The monograph 3 summarizes many classical results regarding $j(\tau)=j_{1}(\tau)$, meaning $N=1$, for an arbitrary CM point $\tau$. Specifically, it is proven that $j(\tau)$ is an algebraic integer and, furthermore, $j(\tau)$ generates the maximal unramified abelian extension of $\mathbb{Q}(\tau)$, often called the Hilbert class field. These results fall within the general framework of Hilbert's twelfth problem, stemming from Kronecker's Jugendtraum, and we refer the interested reader to the article [14] for a fascinating historical discussion.

J. J. acknowledges grant support from PSC-CUNY grants. 
For other genus zero groups $\Gamma_{0}(N)^{+}$, a canonical generator $j_{N}(\tau)$ of the function field came to light as a part of the Monstrous Moonshine conjectures; see [5. The arithmetic nature of $j_{N}(\tau)$ was studied in 4]; in general terms, the main results in [4] are in parallel with the classical setting when $N=1$ as summarized in [3].

However, when $\Gamma_{0}(N)^{+}$has genus one, there is a competing theory of transcendence which somewhat clouds one's expectations. Specifically, let $E_{N}^{+}$denote a fundamental parallelogram for the corresponding elliptic curve (11). Letting $\zeta$ denote the holomorphic coordinate on $E_{N}^{+}$, there exists a biholomorphic map from $Y_{N}^{+}$to $E_{N}^{+}$which sends $i \infty$ from $Y_{N}^{+}$to zero on $E_{N}^{+}$. With this set-up, $x_{N}(\tau)$ is simply the pull-back of the Weierstrass $\wp$-function $\wp_{N}$ on $E_{N}^{+}$, meaning $x_{N}(\tau)=\wp_{N}(\zeta(\tau))$ where, in a slight abuse of notation, we use $\zeta$ to denote the biholomorphic map from $Y_{N}^{+}$to $E_{N}^{+}$. Whereas $y_{N}$ is pull-back of $\wp_{N}^{\prime}$, the differentiation of $\wp_{N}$ is with respect of the coordinate $\zeta$ on $E_{N}^{+}$, not $\tau$ on $\mathbb{H}$. However, one has that

$$
y_{N}(\tau)=\frac{d x_{N}(\tau)}{d \tau}\left(\frac{d \zeta}{d \tau}\right)^{-1} .
$$

In other words, the ratio $x_{N}^{\prime} / y_{N}$, where the prime denotes differentation with respect to $\tau \in \mathbb{H}$, is equal to $d \zeta / d \tau$, the derivative of the biholomorphic map from $Y_{N}^{+}$to $E_{N}^{+}$.

With all this, let us recall the following important corollary of the Schneider-Lang theorem, which we quote from page 22 of [10].

Let $\wp$ be a Weierstrass function with algebraic $g_{2}$ and $g_{3}$. If $\alpha$ is algebraic $\neq 0$, then $\wp(\alpha)$ is transcendental.

In short, if $\Gamma_{0}(N)^{+}$has genus zero, then $j_{N}(\tau)$ for any CM point $\tau \in \mathbb{H}$ is algebraic, but if $\Gamma_{0}(N)^{+}$has genus one, then $\wp_{N}(\alpha)$ is transcendental for any non-zero algebraic $\alpha \in \mathbb{C}$. Additionally, Schneider proved that if $\tau \in \mathbb{H}$ is algebraic but not a CM point, then $j(\tau)$ is transcendental. So, there are various results which point both in the direction of algebraicity and in the direction of transcendence of values $x_{N}(\tau)$ and $y_{N}(\tau)$ for CM points $\tau \in \mathbb{H}$.

The main result of the present article is to derive various results regarding the algebraic nature of $x_{N}(\tau)$ and $y_{N}(\tau)$ for CM points $\tau \in \mathbb{H}$. The nature of the results we obtain are as follows.

Let $Q=[a, b, c]$ with $a>0$ be a primitive positive definite quadratic form with discriminant $b^{2}-4 a c=M^{2} d_{K}<0$, and denote by $\tau$ the complex root of $Q(\tau, 1)=a \tau^{2}+b \tau+c=0$ with $(a, N)=1$ and $\tau \in \mathbb{H}$. Let $K=\mathbb{Q}(\tau)$ be the corresponding imaginary quadratic field of discriminant $d_{K}$. We prove that for every square-free integer $N$ such that the arithmetic group $\Gamma_{0}(N)^{+}$has genus one, the values $x_{N}(\tau)$ and $y_{N}(\tau)$ are algebraic integers. Indeed, for each such $\tau$, we prove that given the value of $x_{N}(\tau)$, the equation (1) which is quadratic in $y_{N}(\tau)$, factors; in other words, the discriminant of (11) as a quadratic in $y_{N}(\tau)$ factors in the field $\mathbb{Q}\left(\tau, x_{N}(\tau)\right)$. Additionally, we also show that $x_{N}(\tau)$ and $y_{N}(\tau)$ are algebraic integers when evaluated at $\tau \in \mathbb{H}$ which is a fixed point of a product $\gamma \omega$, for some $\gamma \in \Gamma_{0}(N)^{+}$and $\omega \in \Omega(m)$, for $m>1$ coprime to $N$, where $\Omega(m)$ is defined by (2) below.

When combining our results with the above-mentioned Schneider-Lang theorem, we arrive at the following conclusion.

The above-described bi-holomorphic map $\zeta$ from $Y_{N}^{+}$to $E_{N}^{+}$sends CM points in $\mathbb{H}$ to transcendental points in $\mathbb{C}$.

Furthermore, assuming that $N$ is prime and $(a, N)=1$, where $(\cdot, \cdot)$ denotes the greatest common divisor, we show that $K\left(x_{N}(\tau)\right)$ generates the ring class field of an imaginary quadratic order $\mathcal{O}^{\prime}$ in $K=\mathbb{Q}(\tau)$ of discriminant $(M N)^{2} d_{K}$. In the case when $(a, N)>1$, we give some explicit computations of generating polynomials for $x_{N}(\tau)$. Our examples yield instances when $K\left(x_{N}(\tau)\right)$ is a subfield of the class field. In section 4 we present instances of different situations, identifying certain ring class fields and subfields of the ring class fields.

Further arithmetic corollaries and possible questions of future study are presented in the concluding section.

As with similar problems, our proof that $x_{N}(\tau)$ and $y_{N}(\tau)$ are algebraic integers for CM points hinges on the construction of modular polynomials. In the study of the singular moduli associated to $j$, modular polynomials tend to have very large coefficients, which stems from the size of the coefficients of $j$ in its $q$-expansion. The elliptic curve primality proving (ECPP) algorithm developed in [1] utilizes the computation of the minimal polynomial of a generator of the Hilbert class field of a certain imaginary quadratic field. As stated in the MathSciNet review of [17, F. Morain wrote that the "search for an optimal (with respect to the size of the coefficients of its minimal polynomial) invariant is an important one." Empirically, we observed in 8 , that the coefficients in the $q$-expansion of $x_{N}$ and $y_{N}$ tend to decrease as $N$ increases. Specifically, in Example 10 below, we compute the minimal polynomials for both $j$ and $x_{37}$ at $\tau=i \sqrt{2 / 37}$. In this instance, the minimal polynomial for $j(i \sqrt{74})$ has coefficients as large as 
$6.6 \times 10^{73}$, whereas the minimal polynomial for $x_{N}(i \sqrt{2 / 37})$ has coefficients less than or equal to 21904 . In other words, one practical application of our results seems to be the ability to construct modular polynomials for Hilbert class fields, and its subfields, which have much smaller coefficients than the corresponding polynomials which are constructed using $j(\tau)$, and perhaps other modular functions.

Several computations within the present paper were obtained with computer aided algebra which employ and extend computations developed in [8. All computer codes, programs, and output will be made publicly available.

\section{Preliminaries}

2.1. Basic notation. Throughout this article, $N$ will denote a positive and square-free integer. For any positive integer $m$, let us denote by $\sigma_{1}(m)$ the sum of positive divisors $m$, and put

$$
\sigma_{1}^{+}(m)=\sum_{d \mid m} \max \{d, m / d\}
$$

The group $\Gamma_{0}(N)^{+}$is defined above. Let $\Gamma_{0}(N)^{\dagger}$ denote the arithmetic group by

$$
\Gamma_{0}(N)^{\dagger}=\Gamma_{0}(N) \cup \Gamma_{0}(N) \gamma_{N}
$$

where $\Gamma_{0}(N)$ is the Hecke congruence subgroup of level $N$, and

$$
\gamma_{N}=\left(\begin{array}{cc}
0 & -1 / \sqrt{N} \\
\sqrt{N} & 0
\end{array}\right)
$$

is the Fricke involution. Clearly, $\Gamma_{0}(N)^{\dagger} \subseteq \Gamma_{0}(N)^{+}$with equality if and only if $N$ is prime.

We let $\mathbb{H}$ denote the hyperbolic upper half plane, with global coordinate $z$ and $q$-parameter $q=e^{2 \pi i z}$. As above, $\tau$ will denote a CM point. If we are considering a function on the upper half plane, its argument will be $z$, and if we are considering its special value at a CM point, its argument will be $\tau$. Discrete groups such as $\Gamma_{0}(N)^{+}$act on $\mathbb{H}$ through fractional linear transformations.

2.2. Properties of the group $\Gamma_{0}(N)^{+}$. Following the notation of [4], for any positive integer $m$ we put

$$
\Omega(m)=\left\{\left(\begin{array}{ll}
a & b \\
0 & d
\end{array}\right): a, b, d \in \mathbb{Z}, d>0, a d=m,(a, b, d)=1,0 \leq b<d\right\} .
$$

The cardinality of $\Omega(m)$ is denoted by $\psi(m)$. The following proposition collects various statements of [4]; see, in particular, Lemma 2.1, Lemma 2.3, and the proof of part (1) of Theorem 2.6.

Proposition 1 (4]). Let $N$ be a square-free number and let $m$ be a positive integer such that $(m, N)=1$. Then, for all $\omega \in \Omega(m)$, the following results hold:

(i) The sets $\Gamma_{0}(N)^{+} \omega$ are disjoint;

(ii) The sets $\Gamma_{0}(N)^{+} \omega$ are permuted through right multiplication by elements $\eta$ of $\Gamma_{0}(N)^{+}$;

(iii) The group $\Gamma_{0}(N)$ acts transitively on the sets $\Gamma_{0}(N) \omega$ by multiplication on the right.

We also will use the following result; see Lemma 3.2 of [4.

Lemma $2\left([4)\right.$. Let $Q=[a, b, c]$ be a primitive positive definite quadratic form. Then, $Q(x, y)=a x^{2}+b x y+c y^{2}$ with $x, y \in \mathbb{Z}$ represents infinitely many primes.

2.3. Shimura reciprocity law. We will recall now a special case of general Shimura reciprocity law for modular functions of level $N$; see [15] and [11]. For a proof of the statement, we refer to subsection (3.7.3) of [4].

Let $\mathrm{GL}^{+}(2, \mathbb{Q})$ denotes the set of $2 \times 2$ matrices with rational entries and positive determinant. Let $f$ be a modular function of level $N$ with rational Fourier coefficients, and let $f(\tau)^{\mathfrak{a}}$ denote the action of the element of the Galois group corresponding to the ideal $\mathfrak{a}$; see 4 for a more detailed explanation of this action.

Proposition 3. Let $\tau \in \mathbb{H}$ be a $C M$ point, and set $K=\mathbb{Q}(\tau)$. Then for any ideal $\mathfrak{a}$ in the ray class group $J_{K}^{N}$ with modulus $N$, there exists a matrix $\mathcal{A} \in \mathrm{GL}^{+}(2, \mathbb{Q})$ such that $f(\tau)^{\mathfrak{a}}=f(\mathcal{A} \tau)$. 
If $f$ is a modular function on $\Gamma_{0}(N)^{+}$, then for all $z \in \mathbb{H}$ and $\gamma \in \Gamma_{0}(N)$, we have that $f(\gamma z)=f(z)$. Moreover $f\left(W_{e} z\right)=f(z)$ for all $e \mid N$, where $W_{e}$ is a matrix with integer entries, determinant $e$, diagonal elements divisible by $e$, and left-lower entry divisible by $N$. Let $\Gamma_{0}(N, e)$ denote the set of all such matrices, noting that $\Gamma_{0}(N, 1)=\Gamma_{0}(N)$. Then, it is elementary to show that $\mathrm{GL}^{+}(2, \mathbb{Q})=\cup_{e \mid N} \Gamma_{0}(N, e) \mathrm{GL}^{+}(2, \mathbb{Q})$. Additionally, for $\mathcal{A} \in \mathrm{GL}^{+}(2, \mathbb{Q})$, one has $f(\mathcal{A} z)=f(z)$ if and only if $\mathcal{A} \in \cup_{e \mid N} \Gamma_{0}(N, e) \mathrm{GL}^{+}(2, \mathbb{Q})_{z}$ and where $\mathrm{GL}^{+}(2, \mathbb{Q})_{z}$ denotes the isotropy subgroup at $z$.

The significance of Shimura reciprocity in explicit class field theory lies in the fact that, as described in 15, the element of the Galois group corresponding to the ideal $\mathfrak{a}$ lies in the fixing group of $f(z)$ if and only if $f(\mathcal{A} z)=f(z)$.

\section{Algebraicity of Values of Generators}

When the CM point $\tau \in \mathbb{H}$ is non-elliptic, the proof of algebraicity of $x_{N}(z)$ and $y_{N}(z)$ which we develop below is closely modelled on classical proofs; see, for example, the argument in 18 and its modification in [4. One significant difference, however, which appears since $g \geq 1$ is the fact that one has two generators for the function field. Consequently, any modular polynomial is a polynomial in two variables. In order to address this addition level of complication, one needs to exploit both the cubic equation (1) which relates the two generators, as well as the established result that each generator has a monic $q$-expansion with integral coefficients.

We will follow the notation of [8], where the generators of the function fields associated to $\Gamma_{0}(N)^{+}$are denoted by $x_{N}$ and $y_{N}$.

We begin with the following proposition.

Proposition 4. Let $N$ be a square-free number, $m>1$ an integer such that $(m, N)=1$, and define the polynomial

$$
\Phi_{m, N}(X)=\prod_{\omega \in \Omega(m)}\left(X-x_{N} \circ \omega\right) .
$$

Then, $\Phi_{m, N}(X) \in \mathbb{Z}\left[X, x_{N}, y_{N}\right]$. Moreover, if $m$ is not a perfect square, there exist polynomials $P_{m, N}(x)$ and $Q_{m, N}(x)$ in $\mathbb{Z}[x]$ such that

$$
\Phi_{m, N}\left(x_{N}(z)\right)=P_{m, N}\left(x_{N}(z)\right)+y_{N}(z) Q_{m, N}\left(x_{N}(z)\right) .
$$

The degree of the polynomial $P_{m, N}(x)$ is $\sigma_{1}^{+}(m)$ and its lead coefficient is \pm 1 . The degree of the polynomial $Q_{m, N}(x)$ is at most $\sigma_{1}^{+}(m)-2$.

Proof. We can write

$$
\Phi_{m, N}(X)=X^{\psi(m)}+\sum_{j=0}^{\psi(m)-1} E_{j, m, N} X^{j},
$$

where $E_{j, m, N}$ are elementary symmetric polynomials in $x_{N} \circ \omega$. By Proposition 1, part (ii), we deduce that each $E_{j, m, N}$ is invariant with respect to $\Gamma_{0}(N)^{+}$. Moreover, when letting $c_{n, N}$ denote the coefficients in the $q$-expansion of $x_{N}(z)$, we see that

$$
\Phi_{m, N}(X)(q)=\prod_{\substack{d d=m \\ d>0}} \prod_{\substack{b \\(b, a, d)=1}}\left(X-\sum_{n=-2}^{\infty} c_{n, N} \zeta_{d}^{b n} q^{a n / d}\right),
$$

where $\zeta_{d}=\exp (2 \pi i / d)$ is the primitive $d$-th root of unity. Since $x_{N}$ has a monic $q$-expansion with integer coefficients and which begins with $q^{-2}$, we have that $c_{n, N} \in \mathbb{Z}$ and $c_{-2, N}=1$. Therefore, each $E_{j, m, N}$ is meromorphic in the cusp at $i \infty$, so then $E_{j, m, N}$ is a modular function on $\Gamma_{0}(N)^{+}$.

From (4), it is immediate that the coefficients in the $q$-expansion of each $E_{j, m, N}$ belong to $\mathbb{Z}\left(\zeta_{m}\right)$. We now argue as from page 69 of [18. The action by the Galois conjugation $\zeta_{d} \mapsto \zeta_{d}^{r}$ with $r \in(\mathbb{Z} / d \mathbb{Z})^{*}$ results in replacing $b$ in the inner product of (4) with $r b$, which runs over the same set. Therefore, the coefficients in the inner product are Galois invariant, hence are rational integers. By Proposition[1(ii), the product over $b$ is invariant under translation $z \mapsto z+1$. Therefore, the $q$-expansion on the right hand side of (4) has integer coefficients and integer powers of $q$. Consequently, each $E_{j, m, N}$ has a $q$-expansion with integer coefficients.

Since $x_{N}$ and $y_{N}$ generate the function field associated to $\Gamma_{0}(N)^{+}$, there exist polynomials $F_{j, m, N}(x, y)$ with rational coefficients such that $E_{j, m, N}=F_{j, m, N}\left(x_{N}, y_{N}\right)$. Recall that the $q$-expansion of $x_{N}$ begins with $q^{-2}$ and 
has integer coefficients, while the $q$-expansion of $y_{N}$ begins with $q^{-3}$ and has integer coefficients. One can then equate the coefficients in the various $q$-expansions to conclude that the coefficients of $F_{j, m, N}(x, y)$ are integers, which proves the first statement of the theorem.

Let us now set $X=x_{N}(z)$ and substitute in (4). By arguing analogously as in [18, Proposition 24, we deduce that for non-square $m$ one has that

$$
\Phi_{m, N}\left(x_{N}(z)\right) \sim \pm q^{-2 \sigma_{1}^{+}(m)}, \quad \text { as } \operatorname{Im}(z) \rightarrow \infty .
$$

Therefore, $\Phi_{m, N}\left(x_{N}(z)\right)$ is a modular function on $\Gamma_{0}(N)^{+}$with a pole of order $2 \sigma_{1}^{+}(m)$ at $i \infty$. Since $x_{N}$ and $y_{N}$ are generators of the function field, which satisfy the cubic equation (1), we can eliminate all powers of $y_{N}$ greater than one and obtain equation (3).

Finally, we complete the proof by using that $x_{N}$ and $y_{N}$ have $q$-expansions with lead term $q^{-2}$ and $q^{-3}$, respectively, which determines the degree and the lead coefficient of $P_{m, N}(x)$ and bounds the degree of $Q_{m, N}(x)$.

We are now in position to prove the analogue of 4, Proposition 3.3 which asserts that Hauptmodli for genus zero groups $\Gamma_{0}(N)^{+}$, when properly normalized, and evaluated at certain CM points are algebraic integers. In fact, we will prove a slightly more general result, allowing for the CM points $\tau$ which are zeros of an equation $a z^{2}+b z+c=0$ with $(a, N)>1$, but requiring additional properties.

Proposition 5. Let $K$ be an imaginary quadratic field of discriminant $d_{K}$. Let $\mathbf{T}\left(\tau_{0}\right)$ and $\mathbf{N}\left(\tau_{0}\right)$ denote the trace and the norm of an algebraic integer $\tau_{0}$ in $\mathcal{O}_{K}$, the ring of integers of $K$, from $K$ to $\mathbb{Q}$. Assume that either of the following two conditions hold:

i) The point $\tau_{0} \in \mathcal{O}_{K}$ is a root of a quadratic equation $z^{2}+\mathbf{T}\left(\tau_{0}\right) z+\mathbf{N}\left(\tau_{0}\right)=0$ with $\mathbf{T}\left(\tau_{0}\right)^{2}-4 \mathbf{N}\left(\tau_{0}\right)=d_{K}$;

ii) There exists an integer $m>1$, not a perfect square and coprime to $N$ and such that $\tau_{0}=\gamma \omega \tau_{0}$ for some $\gamma \in \Gamma_{0}(N)^{+}$and $\omega \in \Omega(m)$.

Then both $x_{N}\left(\tau_{0}\right)$ and $y_{N}\left(\tau_{0}\right)$ are algebraic integers.

Proof. Let us first prove (i). Following the method of the proof of 4, Proposition 3.3 and applying Lemma 2, we conclude there exists a prime $p$ such that $\tau_{0}=\rho \tau_{0}$ where $\rho$ is a primitive matrix of determinant $p$. Proposition 1 implies that $\rho=\gamma \omega$ for some $\gamma \in \Gamma_{0}(N)$ and $\omega \in \Omega(p)$. By taking $X=x_{N} \circ \omega\left(\tau_{0}\right)$, we have that $\Phi_{p, N}\left(x_{N}\left(\tau_{0}\right)\right)=0$. By combining with (3), we arrive at the expression

$$
y_{N}\left(\tau_{0}\right)=-\frac{P_{p, N}\left(x_{N}\left(\tau_{0}\right)\right)}{Q_{p, N}\left(x_{N}\left(\tau_{0}\right)\right)} .
$$

Recall that $P_{p, N}(x), Q_{p, N}(x) \in \mathbb{Z}[x]$ are polynomials of degrees $\sigma_{1}^{+}(p)$ and $\sigma_{1}^{+}(p)-2$, respectively, and the lead coefficient in $P_{p, N}(x)$ is \pm 1 .

Since $x_{N}(z)$ and $y_{N}(z)$ satisfy the cubic equation (1), we combine with (5) and, after multiplying by $Q_{p, N}\left(x_{N}\left(\tau_{0}\right)\right)^{2}$, obtain the equation

$$
\begin{array}{r}
P_{p, N}\left(x_{N}\left(\tau_{0}\right)\right)^{2}-Q_{p, N}\left(x_{N}\left(\tau_{0}\right)\right)^{2} x_{N}\left(\tau_{0}\right)^{3}+A_{N} P_{p, N}\left(x_{N}\left(\tau_{0}\right)\right) Q_{p, N}\left(x_{N}\left(\tau_{0}\right)\right) x_{N}\left(\tau_{0}\right)-B_{N} Q_{p, N}\left(x_{N}\left(\tau_{0}\right)\right)^{2} x_{N}\left(\tau_{0}\right)^{2} \\
+C_{N} P_{p, N}\left(x_{N}\left(\tau_{0}\right)\right) Q_{p, N}\left(x_{N}\left(\tau_{0}\right)\right)-D_{N} Q_{p, N}\left(x_{N}\left(\tau_{0}\right)\right)^{2} x_{N}\left(\tau_{0}\right)-E_{N} Q_{p, N}\left(x_{N}\left(\tau_{0}\right)\right)^{2}=0 .
\end{array}
$$

In other words, $x_{N}\left(\tau_{0}\right)$ is a root of a monic polynomial of degree $4 p$ with integer coefficients. Therefore, $x_{N}\left(\tau_{0}\right)$ is an algebraic integer.

As for $y_{N}\left(\tau_{0}\right)$, note that from (11) we immediately get that $y_{N}\left(\tau_{0}\right)$ is an algebraic integer if $x_{N}\left(\tau_{0}\right)$ is an algebraic integer since one can solve for $y_{N}\left(\tau_{0}\right)$ given the value of $x_{N}\left(\tau_{0}\right)$ using the quadratic formula. This completes the proof of the theorem assuming (i).

Let us now assume (ii) is true. Obviously, $x_{N}\left(\gamma\left(\omega\left(\tau_{0}\right)\right)\right)=x_{N}\left(\omega\left(\tau_{0}\right)\right)=x_{N}\left(\tau_{0}\right)$. Hence, we take $X=x_{N} \circ \omega\left(\tau_{0}\right)$ and repeat the proof above, obtaining a degree $2 \sigma_{1}^{+}(m)$ monic polynomial with integer coefficients whose root is $x_{N}\left(\tau_{0}\right)$. One can continue, analogously as in the proof assuming condition (i) to conclude that $x_{N}\left(\tau_{0}\right)$ and $y_{N}\left(\tau_{0}\right)$ are algebraic integers.

Remark 6. As stated above, given the value of $x_{N}\left(\tau_{0}\right)$, one can solve for $y_{N}\left(\tau_{0}\right)$ using (1) and the quadratic formula. Since $x_{N}\left(\tau_{0}\right)$ is an algebraic integer, then $y_{N}\left(\tau_{0}\right)$ is also an algebraic integer, though possibly in a quadratic extension 
of a field containing $x_{N}\left(\tau_{0}\right)$. However, by (5), we have that $y_{N}\left(\tau_{0}\right)$ lies in the same field as $x_{N}\left(\tau_{0}\right)$. In other words, for any CM point $\tau_{0}$, we have shown that $x_{N}\left(\tau_{0}\right)$ is an algebraic integer. Furthermore, the discriminant of the quadratic equation (11) in $y_{N}\left(\tau_{0}\right)$ is a square in the field in which $x_{N}\left(\tau_{0}\right)$ lies.

The following theorem is the the main result in this section.

Theorem 7. Let $N$ be a square-free number such that $\Gamma_{0}(N)^{+}$has genus one.

i) Let $Q=[a, b, c]$ be a primitive positive definite quadratic form of discriminant $d_{K}$ such that $(a, N)=1$. Then $x_{N}(\tau)$ and $y_{N}(\tau)$ are algebraic integers for every root $\tau \in \mathbb{H}$ of the quadratic equation $Q(z, 1)=0$.

ii) If $\tau \in \mathbb{H}$ is root of the quadratic equation $Q(z, 1)=0$ such that $(a, N)>1$, but there exists an integer $m>1$, not a perfect square and coprime to $N$ and such that $\tau=\gamma \omega \tau$ where $\omega \in \Omega(m)$ and $\gamma \in \Gamma_{0}(N)^{+}$, then $x_{N}(\tau)$ and $y_{N}(\tau)$ are algebraic integers.

Proof. The proof of part (i) follows the lines of the proof of 4, Theorem 3.4. By applying Proposition 1, we can write $\tau=\omega_{0} \tau_{0}$ for some $\omega_{0} \in \Omega(a)$ and $\tau_{0} \in \mathcal{O}_{K}$. By assumption, we have that $(a, N)=1$, and by Proposition 4 the polynomial $\Phi_{a, N}(X)$ has coefficients in $\mathbb{Z}\left[x_{N}, y_{N}\right]$. In other words, $x_{N}(\tau)=x_{N}\left(\omega_{0}\left(\tau_{0}\right)\right)$ is integral over $\mathbb{Z}\left[x_{N}\left(\tau_{0}\right), y_{N}\left(\tau_{0}\right)\right]$, which together with Proposition 5 yields the proof of (i).

The proof of (ii) follows directly from Proposition 5 (ii).

Remark 8. Note that Theorem 7 (ii) also holds true when the square-free level $N$ is such that the genus of the surface $X_{N}=\overline{\Gamma_{0}(N)^{+}} \backslash \mathbb{H}$ is zero. Namely, in this case, denoting by $j_{N}(z)$ the Hauptmodul on $\Gamma_{0}(N)^{+}$, according to Proposition 2.5. of [4, for integer $m>1$, not a perfect square and coprime to $N$, the modular polynomial $\Phi_{m}(X)=\prod_{\omega \in \Omega(m)}\left(X-j_{N} \circ \omega\right)$ belongs to $\mathbb{Z}\left[X, j_{N}\right]$. Therefore, for $\tau \in \mathbb{H}$, such that $\tau=\gamma \omega \tau$, and for some $\gamma \in \Gamma_{0}(N)^{+}$with $\omega \in \Omega(m)$, we immediately deduce that $j_{N}(\tau)$ is a zero of a monic polynomial with integer coefficients of degree $\sigma_{1}^{+}(m)$. Hence, $j_{N}(\tau)$ is an algebraic integer.

This case was not covered by Theorem 3.4. of [4, but follows immediately from their results.

\section{Examples: Modular Polynomials FOR SMAll ORDers}

As before, assume that $N$ is a square-free integer such that $\Gamma_{0}(N)^{+}$has genus one. Choose $m$ to be a prime number so that $m \nmid N$. We will consider small values of $m$. Recall that if $m$ is prime then $\sigma_{1}^{+}(m)=2 m$. With these assumptions, we have that

$$
\Phi_{m, N}(X)(z)=\left(X-x_{N}(m z)\right) \prod_{j=0}^{m-1}\left(X-x_{N}\left(\frac{z+j}{m}\right)\right) .
$$

Recall that $x_{N}$ has a $q$-expansion of the form

$$
x_{N}(z)=q^{-2}+c_{-1, N} q^{-1}+\sum_{n=1}^{\infty} c_{n, N} q^{n}
$$

with the coefficients $c_{n, N}$ that were explicitly computed in [8, 9]. By substituting the $q$-expansion for $x_{N}$ into (6), we obtain the $q$-expansion of the modular polynomial $\Phi_{m, N}(X)$. By further replacing $X$ by $x_{N}(z)$ in (6) and comparing the coefficients in the $q$-expansions on both sides of (3), one obtains the polynomials $P_{m, N}$ and $Q_{m, N}$ which were derived in Proposition 5. Numerous examples of the polynomials $P_{m, N}$ and $Q_{m, N}$ were computed and are listed in Appendix $\mathrm{A}$.

In principle, we could take $z$ to be any CM point, which as before we denote by $\tau$; however, in practice it would be difficult to find a corresponding prime $m$. This issue will be treated by separate examples. Computationally, it is desirable to choose a small or moderate prime $m$, always coprime to $N$, and take one of the matrices of $\Omega(m)$ to be such that the quadratic equation $\gamma \omega z=z$ has negative discriminant for $\gamma \in \Gamma_{0}(N)^{+}$and $\omega \in \Omega(m)$. One then solves for the fixed points of the transformations $\gamma \omega$. For each such fixed point $\tau$, one has that $\Phi_{m, N}\left(x_{N}(\tau)\right)=0$. Therefore, $x_{N}(\tau)$ is a zero of a monic polynomial

$$
\begin{aligned}
P_{m, N}(x)^{2}-Q_{m, N}(x)^{2} x^{3}+A_{N} P_{m, N}(x) Q_{m, N} & (x) x-B_{N} Q_{m, N}(x)^{2} x^{2} \\
& \left.+C_{N} P_{m, N}(x) Q_{m, N}(x)\right)-D_{N} Q_{m, N}(x)^{2} x-E_{N} Q_{m, N}(x)^{2}=0 .
\end{aligned}
$$


Equation (7) is a generating polynomial for $x_{N}(\tau)$. The generating polynomial is computable for each fixed $N$ and $m$ by the manner described above.

As an example, consider

$$
\omega_{m}=\left(\begin{array}{cc}
m & 0 \\
0 & 1
\end{array}\right) \quad \text { or } \quad \omega_{m}=\left(\begin{array}{cc}
1 & 0 \\
0 & m
\end{array}\right) \quad \text { and } \quad \gamma=\gamma_{N}=\left(\begin{array}{cc}
0 & -1 / \sqrt{N} \\
\sqrt{N} & 0
\end{array}\right) .
$$

Then the quadratic equation $\gamma_{N} \omega_{m} z=z$ has negative discriminant $D=-4 m N$ and the corresponding CM points are

$$
\tau=i \sqrt{\frac{1}{N m}} \text { and } \tau=i \sqrt{\frac{m}{N}},
$$

respectively. Since both CM points are $\Gamma_{0}(N)^{+}$-equivalent, it is enough to consider only the latter point.

For all square-free positive $N$ such that $\Gamma_{0}(N)^{+}$has genus one and some small primes $m$, the generating polynomials for $x_{N}\left(i \sqrt{\frac{m}{N}}\right)$ are given in Appendix B. In Appendix C we list the numerical values of the generators $x_{N}$ and $y_{N}$ at the CM point $\tau=i \sqrt{m / N}$ for various $m$ and $N$.

The generating polynomials of $x_{N}\left(i \sqrt{\frac{m}{N}}\right)$ are not necessarily irreducible. After factoring the generating polynomials, and by inspection of finite number of cases presented in Appendices B and [C] one can compare the approximate values of $x_{N}$ at the CM points $\tau=i \sqrt{m / N}$ with the zeros of the irreducible factors of the generating polynomials of $x_{N}\left(i \sqrt{\frac{m}{N}}\right)$. In this manner, one then is able to deduce the irreducible generating polynomials of $x_{N}\left(i \sqrt{\frac{m}{N}}\right)$. In the examples developed below, these polynomials generate real subfields of the class field of the order $\mathcal{O}_{K}=\mathbb{Z}+\sqrt{-(m N)} \mathbb{Z}$ associated to the imaginary quadratic number field $K=\mathbb{Q}(\sqrt{-(m N)})$. Moreover, the above construction may be derived for an arbitrary $m$ coprime to $N$, in which case the modular polynomial $\Phi_{m, N}$ certainly would be more involved.

In general, we have the following proposition.

Proposition 9. Let $N$ be a square-free number as above. Let $m>1$ and $M \geq 1$ be two square-free coprime numbers such that $m M$ is coprime to $N$. Then $\mathbb{Q}\left(x_{N}\left(i M \sqrt{\frac{m}{N}}\right)\right)$ is the real subfield of the class field of the order $\mathcal{O}_{K, M}=\mathbb{Z}+M \sqrt{-(m N)} \mathbb{Z}$ associated to the imaginary quadratic number field $K=\mathbb{Q}(\sqrt{-(m N)})$. Moreover, the field $K\left(x_{N}\left(i M \sqrt{\frac{m}{N}}\right)\right)$ is a subfield of the ring class field of the order $\mathcal{O}_{K, M}=\mathbb{Z}+M \sqrt{-(m N)} \mathbb{Z}$ over $K$.

Proof. According to the results in classical complex multiplication and class field theory, see e.g. [6], the field $K\left(j\left(i M \sqrt{\frac{m}{N}}\right)\right)$ is the class field of the order $\mathcal{O}_{K, M}$ over $K$ of discriminant $-4 M^{2} m N$. The function $x_{N}(z)$ is a modular function on $\Gamma_{0}(N)$. Furthermore, according to [6], Theorem 11.9. $x_{N}(z)$ is a rational function of $j(z)$ and $j(N z)$. Therefore, $x_{N}\left(i M \sqrt{\frac{m}{N}}\right)$ is a rational function of $j\left(i M \sqrt{\frac{m}{N}}\right)$ and $j\left(i M N \sqrt{\frac{m}{N}}\right)=j(i M \sqrt{m N})$.

On the other hand, $i M \sqrt{m N}$ is a zero of the equation $x^{2}+m M^{2} N=0$ with discriminant equal to $-4 M^{2} m N$. As such, $j(i M \sqrt{m N})$ also generates the class field of the order $\mathcal{O}_{K, M}$ over $K$. With this, we have proven that $x_{N}\left(i M \sqrt{\frac{m}{N}}\right)$ is a rational function of two elements of the class field of the order $\mathcal{O}_{K, M}$. Therefore, $x_{N}\left(i M \sqrt{\frac{m}{N}}\right)$ belongs to $\mathcal{O}_{K, M}$. In other words, the field generated over $\mathbb{Q}$ by $x_{N}\left(i M \sqrt{\frac{m}{N}}\right)$ and $i \sqrt{m N}$ is a subfield of the ring class field of $\mathcal{O}_{K, M}$, and $\mathbb{Q}\left(x_{N}\left(i M \sqrt{\frac{m}{N}}\right)\right)$ is the real subfield of the ring class field $K\left(j\left(i M \sqrt{\frac{m}{N}}\right)\right)$.

Remark 10. In the case when the square-free level $N$ is such that the genus of the surface $X_{N}=\overline{\Gamma_{0}(N)^{+}} \backslash \mathbb{H}$ is zero, the above reasoning may be repeated verbatim to prove that $j_{N}\left(i M \sqrt{\frac{m}{N}}\right)$ generates over $\mathbb{Q}$ a real subfield of the class field of the order $\mathcal{O}_{K, M}=\mathbb{Z}+M \sqrt{-m N} \mathbb{Z}$ of the number field $K=\mathbb{Q}(\sqrt{-m N})$. Note also, that in genus zero case (resp. genus one case), the number $j_{N}\left(i \sqrt{\frac{m}{N}}\right)$ (resp. the numbers $x_{N}\left(i \sqrt{\frac{m}{N}}\right)$ and $\left.y_{N}\left(i \sqrt{\frac{m}{N}}\right)\right)$ are algebraic integers for all $m>1$, not a perfect square and coprime to $N$.

Example 11. Take $m=2$ and $N=37$. The CM point $\tau=i \sqrt{2 / 37}$ is a root of $37 z^{2}+2=0$ of discriminant $-4 \cdot 74$. The generating polynomial of $x_{37}\left(i \sqrt{\frac{2}{37}}\right)$ is

$$
\begin{aligned}
x^{8}+4 x^{7}-556 x^{6}-11724 x^{5}-110853 x^{4}- & 588596 x^{3}-1818476 x^{2}-3066560 x-2190400 \\
= & (4+x)(5+x)^{2}\left(-21904-16428 x-4440 x^{2}-481 x^{3}-10 x^{4}+x^{5}\right) .
\end{aligned}
$$


The value $x_{37}(i \sqrt{2 / 37})$ is a root of the irreducible polynomial $\left(-21904-16428 x-4440 x^{2}-481 x^{3}-10 x^{4}+x^{5}\right)$ over $\mathbb{Q}$. Obviously, $\mathbb{Q}\left(x_{37}(i \sqrt{2 / 37})\right)$ is a degree 5 extension of rationals, and the value $y_{37}(i \sqrt{2 / 37})$ belongs to this extension, as shown by equation (5).

Let us now compare with similar computations for the classical $j$-invariant. The CM point $\tau=i \sqrt{2 / 37}$ generates over $\mathbb{Q}$ the imaginary quadratic field $K=\mathbb{Q}(\sqrt{-74})$ with principal order $\mathcal{O}_{K}=\mathbb{Z}(\sqrt{-74})$. The class number for discriminant $-4 \cdot 74$ is 10 . According to the classical complex multiplication theory, there exists a degree 10 monic and irreducible polynomial over $\mathbb{Q}$, which is a minimal polynomial of $j(i \sqrt{74})$ over $\mathbb{Q}$. Through computer aided computation, this minimal polynomial is given by the equation

$$
\begin{aligned}
\mathcal{M}_{74}(X) & =X^{10}-297590021529144696892272 X^{9} \\
& +162320887755075073090532230331568448 X^{8} \\
& +10833723207526124630181274705783365349945344 X^{7} \\
& +723386799641305659734425943574100626119187174203392 X^{6} \\
& -15114530035213509909886819641017229466515976895827935232 X^{5} \\
& +1616977083082946116052753947160450685516405373648400753885184 X^{4} \\
& +15494958955563981344176689890872586852428892851110732280427970560 X^{3} \\
& +138422647379183478029444656847389271920175189635976728903092562558976 X^{2} \\
& +182855248428762163984052227095736275304389818889530398495800513829797888 X \\
& +65537997861811012957774465106804493369424397836065905853257281566511464448
\end{aligned}
$$

The ring class field, which in this case is the Hilbert class field, of the maximal order $\mathcal{O}_{K}=\mathbb{Z}(\sqrt{-74})$ is given by $L=K(j(i \sqrt{74}))$, which is a degree 10 extension of $K$; see, for example [6], Theorem 9.2.

One can show that the polynomial $p_{37,2}(x)=\left(-21904-16428 x-4440 x^{2}-481 x^{3}-10 x^{4}+x^{5}\right)$ is irreducible over the genus field $K(\sqrt{37})=\mathbb{Q}(\sqrt{37}, \sqrt{-2})$ of $K$, which is a subfield of $L$ and a degree 2 extension of $K$. Therefore, $K\left(x_{37}(i \sqrt{2 / 37}), \sqrt{37}\right)$ is a subfield of $L$ and a degree 10 extension of $K$, meaning that the ring class field $L$ of the maximal order $\mathcal{O}_{K}$ over $K$ is equal to $K\left(x_{37}(i \sqrt{2 / 37}), \sqrt{37}\right)$. In other words, the Hilbert class field of $\mathbb{Q}(\sqrt{-74})$ can be obtained either through adjoining roots of the polynomial $\mathcal{M}_{74}$ or of the polynomials $x^{2}+2$ and $p_{37,2}$.

At this time, we draw the reader's attention to [13. In this well-written article, the authors develop in detail the elliptic curve primality proving (ECPP) algorithm, which utilizes certain minimal polynomials constructed from the classical $j$-invariant. In Remark 5.3.3, the author writes that "replacing $j$ by other functions does not change the complexity of the algorithm, though it is crucial in practice". Specifically, the author refers to the practical usefulness of working with minimal polynomials whose coefficients are as small as possible. In this direction, there is an obvious improvement when comparing $\mathcal{M}_{74}$ to $p_{37,2}$.

Example 12. Take $m=3$ and $N=37$. The CM point $\tau=i \sqrt{3 / 37}$ is a root of $37 z^{2}+3=0$ of discriminant $-4 \cdot 111$. The corresponding imaginary quadratic extension is $K=\mathbb{Q}(\sqrt{-111})$. The generating polynomial is

$$
\begin{aligned}
x^{12}-1372 x^{10}- & 41190 x^{9}-619785 x^{8}-5825280 x^{7}-36892492 x^{6}-161860422 x^{5} \\
& \quad-491879369 x^{4}-1009024188 x^{3}-1314153753 x^{2}-952782930 x-273526200 \\
= & (4+x)(5+x)^{2}(6+x)\left(-4107-2738 x-592 x^{2}-37 x^{3}+x^{4}\right)\left(111+222 x+100 x^{2}+17 x^{3}+x^{4}\right) .
\end{aligned}
$$

The value $x_{37}(i \sqrt{3 / 37})$ is a root of $\left(-4107-2738 x-592 x^{2}-37 x^{3}+x^{4}\right)$.

Unlike the previous example, the polynomial $\left(-4107-2738 x-592 x^{2}-37 x^{3}+x^{4}\right)$ though irreducible over $K$ is, in fact, reducible over the genus field $K(\sqrt{37})=\mathbb{Q}(\sqrt{-3}, \sqrt{37})$. Actually, the polynomial factors over $\mathbb{Q}(\sqrt{37})$ as

$$
\begin{aligned}
p_{37,3}(x)=(-4107-2738 x & \left.-592 x^{2}-37 x^{3}+x^{4}\right) \\
= & -\frac{1}{4}\left(185+37 \sqrt{37}+(37+9 \sqrt{37}) x-2 x^{2}\right)\left(-185+37 \sqrt{37}+(-37+9 \sqrt{37}) x+2 x^{2}\right) .
\end{aligned}
$$

The value $x_{37}(i \sqrt{3 / 37})$ is a root of $185+37 \sqrt{37}+(37+9 \sqrt{37}) x-2 x^{2}$, and we obtain the explicit evaluation 


$$
x_{37}(i \sqrt{3 / 37})=\frac{1}{4}(37+\sqrt{37}(9+\sqrt{158+26 \sqrt{37}})) .
$$

The class number for the discriminant $-4 \cdot 111$ is 8 . The class field $L$ of the order $\mathbb{Z}+\sqrt{-111} \mathbb{Z}$ in $K=\mathbb{Q}(\sqrt{-111})$ is a degree 8 abelian extension of $K$. Classically, we have that $L=\mathbb{Q}(j(i \sqrt{111}))$. Since the polynomial $p_{37,3}$ is reducible over the genus field, $K\left(x_{37}(i \sqrt{3 / 37})\right)$ is the subfield of the class field of the order $\mathcal{O}_{K}=\mathbb{Z}+\sqrt{-111} \mathbb{Z}$ such that $\left[L: K\left(x_{37}(i \sqrt{3 / 37})\right)\right]=2$. We then have the sequence of fields

$$
\mathbb{Q}(\sqrt{-111})=K \subset K(\sqrt{37}) \subset K\left(x_{37}(i \sqrt{3 / 37})\right) \subset L=\mathbb{Q}(j(i \sqrt{111}))
$$

where each field is a degree 2 and unramified extension of its precedent.

The above example shows us that the generating polynomial of $x_{N}$ evaluated at a certain CM point enables us to compute different subfields of the ring class field. The following example further illustrates this point,.

Example 13. Take $m=7$ and $N=37$. The CM point $\tau=i \sqrt{7 / 37}$ is a root of the equation $37 z^{2}+7=0$ of discriminant $-4 \cdot 259$. The corresponding imaginary quadratic extension is $K=\mathbb{Q}(\sqrt{-259})$. In this case $x_{37}(i \sqrt{7 / 37})$ is a root of the degree 6 polynomial

$$
p_{37,7}(x)=-998001-961038 x-369297 x^{2}-70596 x^{3}-6671 x^{4}-242 x^{5}+x^{6},
$$

which is a factor of the modular polynomial associated to $m=7$ and $N=37$. The class number of discriminant $-4 \cdot 259$ is 12 . A simple computation shows that $p_{37,7}(x)$ is irreducible over $K$. Hence, $K\left(x_{37}(i \sqrt{7 / 37})\right)$ generates a degree 6 extension of $K$, which is a degree 2 subfield of the ring class field $L$ of the order $\mathcal{O}_{K}=\mathbb{Z}+\sqrt{-259} \mathbb{Z}$ in $K$. The genus field $G$ of $K$ is given by $G=K(\sqrt{-7}, \sqrt{37})=\mathbb{Q}(\sqrt{-7}, \sqrt{37})$. The polynomial $p_{37,7}(x)$ is reducible over $\mathbb{Q}(\sqrt{37})$, factoring as

$$
\begin{aligned}
p_{37,7}(x)=\left(-5994-999 \sqrt{37}-(1702+289 \sqrt{37}) x-(121+22 \sqrt{37}) x^{2}+x^{3}\right) \\
\cdot\left(-5994+999 \sqrt{37}+(-1702+289 \sqrt{37}) x+(-121+22 \sqrt{37}) x^{2}+x^{3}\right) .
\end{aligned}
$$

Therefore, $\mathbb{Q}\left(\sqrt{37}, x_{37}(i \sqrt{7 / 37})\right)$ is a real subfield of the ring class field $L$ of index 4 .

This way, we have constructed two subfields of $L$ : The subfield $K\left(x_{37}(i \sqrt{7 / 37})\right)$ of index 2 , and the real subfield $\mathbb{Q}\left(\sqrt{37}, x_{37}(i \sqrt{7 / 37})\right)$ of index 4 .

Example 14. Take $m=2$ and $N=143$. The CM point $\tau=i \sqrt{2 / 143}$ is a root of the equation $143 z^{2}+2=0$ of discriminant $-4 \cdot 286$ with the class number 12 . The corresponding imaginary quadratic extension is $K=\mathbb{Q}(\sqrt{-286})$. In this case, the generating polynomial for $x_{143}(i \sqrt{2 / 143})$ is

$$
x^{8}-4 x^{7}-8 x^{6}+11 x^{4}=(-1+x) x^{4}\left(-11-11 x-3 x^{2}+x^{3}\right) .
$$

The value $x_{143}(i \sqrt{2 / 143})$ is the real root of $p_{143,2}=-11-11 x-3 x^{2}+x^{3}$, from which we get the evaluation that

$$
x_{143}(i \sqrt{2 / 143})=1+\frac{1}{3} \sqrt[3]{324-6 \sqrt{858}}+\frac{\sqrt[3]{2(54+\sqrt{858})}}{3^{2 / 3}}
$$

The polynomial $p_{143,2}$ is irreducible over both $K$ and the genus field $G=K(\sqrt{-11}, \sqrt{13})=\mathbb{Q}(\sqrt{-11}, \sqrt{13})$. Hence, $K\left(x_{143}(i \sqrt{2 / 143})\right)$ (resp. $G\left(x_{143}(i \sqrt{2 / 143})\right)$ ) is a subfield of the ring class field of the order $\mathcal{O}_{K}=\mathbb{Z}+\sqrt{-286} \mathbb{Z}$ in $K$ of index 4 (resp. 2).

It is possible that the value of $x_{N}$ at the CM point lies in the genus field, as shown by the following example.

Example 15. Take $m=2$ and $N=141$. The CM point $\tau=i \sqrt{2 / 141}$ is a root of the equation $141 z^{2}+2=0$ of discriminant $-4 \cdot 282$. The corresponding imaginary quadratic extension is $K=\mathbb{Q}(\sqrt{-282})$. The generating polynomial for $x_{141}(i \sqrt{2 / 141})$ is

$$
x^{8}-4 x^{7}-8 x^{6}+11 x^{4}=x^{4}(1+x)^{2}\left(3-6 x+x^{2}\right) .
$$

The value $x_{141}(i \sqrt{2 / 141})=3+\sqrt{6}$ is a root of $\left(3-6 x+x^{2}\right)$, which generates a degree two real extension $\mathbb{Q}(\sqrt{6})$. Note that in this case, the genus field of $K=\mathbb{Q}(\sqrt{-282})$ is equal to $K(\sqrt{-2}, \sqrt{6})$, and obviously, $x_{141}(i \sqrt{2 / 141})$ generates over $\mathbb{Q}$ the real subfield of the genus field. 
Recall that $x_{141}$ and $y_{141}$ solve the cubic equation $y^{2}+3 y+\left(3-x^{3}-2 x^{2}\right)=0$. Since $x_{141}(i \sqrt{2 / 141})=3+\sqrt{6}$, we get that $y_{141}(i \sqrt{2 / 141})=6+3 \sqrt{6}$. We can interpret this example as stating that the CM point $\tau=i \sqrt{2 / 141}$ gives rise to the integral point $(3+\sqrt{6}, 6+3 \sqrt{6})$ on the elliptic curve $y^{2}+3 y+\left(3-x^{3}-2 x^{2}\right)=0$ over the field $\mathbb{Q}(\sqrt{6})$.

Example 16. Take $m=2$ and $N=155$. The minimal polynomial is

$$
x^{8}-4 x^{7}-4 x^{6}-8 x^{5}-11 x^{4}-4 x^{3}-6 x^{2}=x^{2}\left(1+x^{2}\right)^{2}\left(-6-4 x+x^{2}\right) .
$$

The value $x_{155}(i \sqrt{2 / 155})=2+\sqrt{10}$ generates $\mathbb{Q}(\sqrt{10})$. From data derived in [8], the corresponding cubic equation is $y^{2}+3 y-x^{3}-2 x^{2}+2=0$. Therefore, we obtain that $y_{155}(i \sqrt{2 / 155})=4+2 \sqrt{10}$. In other words, the CM point $\tau=i \sqrt{2 / 155}$ gives rise to the integral point $(2+\sqrt{10}, 4+2 \sqrt{10})$ on the curve $y^{2}+3 y-x^{3}-2 x^{2}+2=0$ over $\mathbb{Q}(\sqrt{10})$.

There are a few points which are common for all of the above examples. Each CM point considered is a purely imaginary root of an equation of the type $N z^{2}+m=0$ with the lead coefficient equal to the level. Hence, the values of generators $x_{N}$ and $y_{N}$ at the CM point are real. Therefore, the monic, irreducible polynomials of degree $d_{m, N}$ with integer coefficients, whose zeros are $x_{N}(\tau)$ evaluated at such CM points, are generating polynomials for real extensions of $\mathbb{Q}$ of degree $d_{m, N}$. Moreover, all fields generated by the values of $x_{N}$ and $y_{N}$ at $i \sqrt{m / N}$ are subfields of the class field of the maximal order of the imaginary quadratic fields $K=\mathbb{Q}(\sqrt{-m N})$.

\section{Further Applications to EXPlicit ClASS Field THEORY}

In this section we prove that values of $x_{N}$ at CM points, which stem from the quadratic equation $a z^{2}+b z+c=0$ where $(a, N)=1$, indeed generate class fields of corresponding orders over imaginary extensions of rationals. The examples presented above show that this is not the case for $(a, N)=N$. Since the arguments presented are actually a slight modification of arguments from [4, we will give a sketch of the proofs, pointing out the differences arising in the case when genus is positive.

Theorem 17. Let $N$ be a prime number such that the group $\Gamma_{0}(N)^{+}$has genus one. Let $\tau$ be a root of the quadratic equation $a z^{2}+b z+c=0$ with $a>0,(a, N)=1,(a, b, c)=1$, and $b^{2}-4 a c=m^{2} d_{K}<0$. Let $K=\mathbb{Q}(\tau)$ be the field of discriminant $d_{K}$, and let $\mathcal{O}$ be the order in $K$ of discriminant $m^{2} d_{K}$. Then $x_{N}(\tau)$ generates the ring class field of an imaginary quadratic order $\mathcal{O}^{\prime}$ of discriminant $(m N)^{2} d_{K}$.

Proof. Begin by observing that $x_{N}(z)$ is a modular function on $\Gamma_{0}(N)$ with integral $q$-expansion. By [16], the values of $x_{N}(\tau)$ belong to the ray class field of $K$ with modulus $N$. We next apply Shimura reciprocity, Proposition 3 , as in [4], to show that $x_{N}(\tau)$ in fact belongs to a smaller field, namely to the ring class field of an imaginary quadratic order $\mathcal{O}^{\prime}$ of discriminant $(m N)^{2} d_{K}$.

By [4, p. 272, the action of an arbitrary prime ideal $\mathfrak{p}$ of $\mathcal{O}_{K}$ on $x_{N}(\tau)$ is represented by the matrix

$$
\mathcal{A}=\left(\begin{array}{cc}
1 & \frac{r m+b}{2} N k l \\
0 & p
\end{array}\right),
$$

where $p$ is a rational prime not dividing $2 a b c m N$ such that $(p)$ splits in $K$ as $(p)=\mathfrak{p p}$ with $\mathfrak{p}=\left[p, \frac{-r+\sqrt{d_{K}}}{2}\right]$ and $s=\left(r^{2}-d_{K}\right) /(4 p) \in \mathbb{Z},(s, N)=1$, and $k$ and $l$ are solutions of congruences

$$
a k \equiv 1 \bmod p \text { and } N l \equiv 1 \bmod p .
$$

Therefore, by Proposition 3 and the subsequent discussion, the prime ideal $\mathfrak{p}$ fixes $x_{N}(\tau)$ if and only if $x_{N}(\mathcal{A} \tau)=$ $x_{N}(\tau)$, which is equivalent to $\mathcal{A} \in \cup_{e \mid N} \Gamma_{0}(N, e) \mathrm{GL}^{+}(2, \mathbb{Q})_{\tau}$. On the other hand, the condition $(a, N)=1$ ensures that $\mathcal{A}$ does not belong to any coset $\Gamma_{0}(N, e) \mathrm{GL}^{+}(2, \mathbb{Q})_{\tau}$ for $e>1$; see [4], pp. 271-272. Hence, $\mathfrak{p}$ fixes $x_{N}(\tau)$ if and only if $\mathcal{A} \in \Gamma_{0}(N) \mathrm{GL}^{+}(2, \mathbb{Q})_{\tau}$, in which case $\mathfrak{p}$ is a principal ideal, say $(\alpha)$ of $\mathcal{O}$.

Since $x_{N}$ is a modular function of level $N$, we have $K(j(\tau)) \subset K\left(x_{N}(\tau)\right)$. Therefore, $x_{N}(\tau)$ is fixed by all ideals in the principal ideal group $P(\mathcal{O})$. Moreover, since $x_{N}(\tau)$ is an algebraic integer, $K\left(x_{N}(\tau)\right)$ corresponds to the ring class field of $K$ consisting of those principal ideals which fix $x_{N}(\tau)$, in the sense of Shimura reciprocity. We have already proven that every prime ideal $\mathfrak{p}$ in $\mathcal{O}_{K}$ fixing $x_{N}(\tau)$ is actually a principal ideal $(\alpha)$ of $\mathcal{O}$. The same arguments as in [4] yield that $\alpha \in \mathcal{O}^{\prime}$ and that the fixing group of $K\left(x_{N}(\tau)\right)$ is the principal ideal group of $\mathcal{O}^{\prime}$. With all this, the proof is complete. 
If one wants to explicitly construct class fields of a fixed CM point using Theorems 17 and 7 the main problem faced with is the construction of the corresponding modular polynomial. The construction can be completed in two steps: First, one writes $\tau=\omega \tau_{0}$ for $\omega \in \Omega(a)$ and $\tau_{0} \in \mathcal{O}_{K}$, and second, one needs to find a prime $p$ which is coprime to $N$ and such that $\tau_{0}=\rho \tau_{0}$ for some $\rho \in \Omega(p)$. The resulting prime $p$ can be very large, even for some very simple $\tau$. Hence, the modular polynomial can have a huge degree and giant coefficients. Such a polynomial can be computationally cumbersome.

As a result, it is desirable to determine another method for deriving small degree modular polynomials for class field generators. Following [4, we derive another means by which one can obtain minimal polynomials. To do so, we require additional notation.

Let $\Gamma=\Gamma_{0}(1)^{+}$denote the full modular group, and let $Q_{D}$ denote the set of all primitive, positive definite quadratic forms of discriminant $D<0$. Following [4, Section 4], we define the map $\phi: Q_{N^{2} d_{K}} / \Gamma \rightarrow Q_{d_{K}} / \Gamma_{0}(N)$ in the following manner.

(1) Assume a positive integer $M$ is given. If $Q=[a, b, c]$ is a primitive, positive definite quadratic form such that $(a, M)=1$, we set $Q^{\prime}=Q$. If $(a, M)>1$, then there exists $\gamma \in \Gamma$ such that $Q^{\prime}=\left[a^{\prime}, b^{\prime}, c^{\prime}\right]=\gamma^{\mathrm{T}}[a, b, c] \gamma$ satisfies $\left(a^{\prime}, M\right)=1$.

(2) Set $Q^{\prime}=\left[a^{\prime}, b^{\prime}, c^{\prime}\right] \in Q_{N^{2} d_{K}}$. From (1), we have $\left(a^{\prime}, 2 N\right)=1$. Let $N=2^{l} N^{\prime}$. Since $N$ is squarefree, $l \in\{0,1\}$. By applying the Chinese Remainder Theorem, we can find $k$ which solves the system of congruences:

$$
\begin{aligned}
& b^{\prime}+2 a^{\prime} k \equiv 0 \bmod N^{\prime}, \\
& b^{\prime}+2 a^{\prime} k \equiv \begin{cases}0 \bmod 2^{l+2} & \text { if } N^{2} d_{K} \equiv 0 \bmod 4 \\
N \bmod 2^{l+2} & \text { if } N^{2} d_{K} \equiv 1 \bmod 4 .\end{cases}
\end{aligned}
$$

The form $Q^{\prime}$ is $\Gamma$-equivalent to the form $Q_{1}=\left[a_{1}, b_{1}, c_{1}\right]=\left[a^{\prime}, b^{\prime}+2 a^{\prime} k, a^{\prime} k^{2}+b^{\prime} k+c^{\prime}\right]=\left[a^{\prime \prime}, N b^{\prime \prime}, N^{2} c^{\prime \prime}\right]$. (3) Define $\phi(Q)=\left[a^{\prime \prime}, b^{\prime \prime}, c^{\prime \prime}\right]$.

Theorem 18. Let $N$ be a prime number such that the group $\Gamma_{0}(N)^{+}$has genus one with function field generators $x_{N}$ and $y_{N}$ as defined above. Let $K$ be an imaginary quadratic field with discriminant $d_{K}$, and let $\mathcal{O}$ be the order of discriminant $N^{2} d_{K}$. For $j=1, \ldots, h(\mathcal{O})$, where $h(\mathcal{O})$ stands for the class number of $\mathcal{O}$, let $Q_{j}$ form a complete set of representatives for $Q_{N^{2} d_{K}} / \Gamma$. Define the polynomial

$$
\mathcal{M}(X)=\prod_{j=1}^{h(\mathcal{O})}\left(X-x_{N}\left(\tau_{\phi\left(Q_{j}\right)}\right)\right),
$$

where $\tau_{Q}$ is the zero in $\mathbb{H}$ of $Q(z, 1)=0$. Then the polynomial $\mathcal{M}(X)$ is the minimal polynomial of $x_{N}\left(\tau_{0}\right)$, where $\tau_{0} \in \mathcal{O}_{K} \cap \mathbb{H}$ is a root of $z^{2}+z+\left(1-d_{K}\right) / 4=0$ if $d_{K} \equiv 1 \bmod 4$, or a root of $z^{2}-d_{K} / 4=0$ if $d_{K} \equiv 0 \bmod 4$. Moreover, $\mathcal{M}(X)$ is a generating polynomial for the ring class field of $\mathcal{O}$ over $K$.

Proof. The proof of this theorem follows the lines of the proof of the same theorem of 4. The main facts used in the proof are fulfilled in our setting, namely that $x_{N}\left(\tau_{0}\right)$ is an algebraic integer and $L=K\left(x_{N}\left(\tau_{0}\right)\right)$ is the ring class field of the order $\mathcal{O}$.

Theorem 18 describes explicitly how to construct the polynomial $\mathcal{M}(X)$. For a fixed prime level $N$ of genus one, the construction of the polynomial is described in the algorithm below.

Step 1. Choose $\tau_{0}$ which is a root of $z^{2}+z+\left(1-d_{K}\right) / 4=0$ if $d_{K} \equiv 1 \bmod 4$, or a root of $z^{2}-d_{K} / 4=0$ if $d_{K} \equiv 0 \bmod 4$. The point $\tau_{0}$ corresponds to $\phi(Q)$, where $Q$ is the identity class in $Q_{N^{2} d_{K}} / \Gamma$.

Step 2. Compute all $h(\mathcal{O})$ inequivalent primitive quadratic forms $Q_{j}$ of discriminant $N^{2} d_{K}$, where $j=1, \ldots, h(\mathcal{O})$. The list of all such forms is produced after entering the discriminant and flag 1 into the web page [12.

Step 3. Compute $\phi\left(Q_{j}\right)=Q_{j}^{\prime \prime}$ for each quadratic form $Q_{j}$ from Step 2. The computation is completed in the manner described in the text preceding the statement of Theorem 18, Essentially, this computation reduces to solving a system of congruences using the Chinese Remainder Theorem.

Step 4. Compute the zeros $\tau_{j}$ of $Q_{j}^{\prime \prime}(z, 1)=0$ for each $Q_{j}^{\prime \prime}$ above.

Step 5. Compute approximate values of $x_{N}\left(\tau_{j}\right)$ for each $\tau_{j}$ from Step 4. 
Step 6. Evaluate approximately the coefficients of the polynomial $\mathcal{M}(X)=\prod_{j=1}^{h(\mathcal{O})}\left(X-x_{N}\left(\tau_{j}\right)\right)$. We know that the coefficients are integers. Once the coefficients are evaluated sufficiently accurate, we can round them to the nearest integers.

In this manner, one can determine specific generating polynomials $\mathcal{M}(x)$. Examples are listed in Appendix $\mathrm{D}$ for various prime levels $N$ and roots $\tau_{0}$. As it was the case in the genus zero setting, such polynomials have very large coefficients and may not be suitable for algebraic applications. Still, the coefficients are comparatively small with respect to the coefficients obtained by the same method using the classical $j$-invariant

\section{Concluding Remarks}

From a different point of view, given an arbitrarily large number $n$, Theorem 18 allows one to construct the ring class field $L_{n}$ of some order in the imaginary quadratic field such that there exist at least $n$ points on the curve (1) with coordinates that are algebraic integers and belong to the field $L_{n}$.

Corollary 19. Let $N$ be a prime number such that $\Gamma_{0}(N)^{+}$has genus one. Then, for an arbitrary number $n$ there exists an abelian Galois extension $L_{n}$ of an imaginary quadratic field such that the curve (1) possesses at least $n$ points whose coordinates are algebraic integers belonging to $L_{n}$.

Proof. The class number of a negative discriminant $D$ tends to infinity as $|D| \rightarrow \infty$. Hence, for a given $n$ and $N$, there exists a discriminant $d_{K}<0$ of a number field $K=\mathbb{Q}\left(\sqrt{d_{K}}\right)$ such that the class number of discriminant $D=N^{2} d_{K}$ is larger than $n$. Let $L_{n}$ be the ring class field of the order in $K$ of discriminant $D$. In the above notation, the points $\left(x_{N}\left(\tau_{\phi\left(Q_{j}\right)}\right), y_{N}\left(\tau_{\phi\left(Q_{j}\right)}\right)\right), j=1, \ldots, h(D)$ are necessarily distinct points on the curve (11). Indeed, if two such points were the same, this would contradict the fact that $x_{N}$ and $y_{N}$ are generators of function fields and that $\phi\left(Q_{j}\right)$ are distinct elements. The algebraic integrality of coordinates follows from Theorem 7 while Theorem 18 implies that $x_{N}\left(\tau_{\phi\left(Q_{j}\right)}\right), y_{N}\left(\tau_{\phi\left(Q_{j}\right)}\right) \in L_{n}$.

As discussed in Remark 6, we find it particularly interesting that if $\tau$ is a CM point, then $y_{N}(\tau)$ lies in the field generated over $\mathbb{Q}$ by $x_{N}(\tau)$. Another manifestation of this observation is the following. If $x_{N}$ is an arbitrary algebraic integer in a number field $K$, then when solving (11) for $y_{N}$, it seems quite unlikely that $y_{N}$ will also lie in $K$. Nonetheless, if $x_{N}=x_{N}(\tau)$ for some CM point $\tau$, then the discriminant of the quadratic equation (1) in the variable $y_{N}$ is a square in the field $K=\mathbb{Q}\left(x_{N}(\tau)\right)$, so then $y_{N}$ also lies in $K$.

Conversely, assume $x_{N}$ is an algebraic integer in an algebraic number field $K$ such that the discriminant of the quadratic (11) in $y_{N}$ is not a square in $K$. Then $x_{N} \neq x_{N}(\tau)$, for any CM point $\tau \in \mathbb{H}$, defined in Theorem 7

Following existing convention, it seems appropriate to call points of the form $\left(x_{N}(\tau), y_{N}(\tau)\right)$ on (1) Heegner points; see [2]. There are a number of interesting questions that could now be asked, such as the density of Heegner points on (10) within the set of all integral points on (1). We hope to turn to such considerations in the future.

\section{REFERENCES}

[1] A. O. L. Atkin and F. Morain, Elliptic curves and primality proving, Math. Comp. 61 (1993), 29-68.

[2] B. J. Birch, Heegner points: the beginnings. in: Heegner points and Rankin L-series, 1-10, Math. Sci. Res. Inst. Publ., 49, Cambridge Univ. Press, Cambridge, 2004.

[3] Seminar on complex multiplication, eds. A. Borel, S. Chowla, C. S. Herz, K. Iwasawa, J. P. Serre, Lecture Notes in Mathematics 21, Springer-Verlag, Berlin-New York, 1966.

[4] I. Chen and N. Yui, Singular values of Thompson series. In Groups, difference sets, and the Monster (Columbus, OH, 1993), 255-326, Ohio State University Mathematics Research Institute Publications, 4, de Gruyter, Berlin, 1996.

[5] J. H. Conway and S. P. Norton, Monstrous moonshine, Bull. London Math. Soc. 11 (1979), 308-339.

[6] D. Cox, Primes of the form $x^{2}+n y^{2}$, J. Wiley and Sons, New York, 1989.

[7] C. J. Cummins, Congruence subgroups of groups commensurable with PSL(2, Z) of genus 0 and 1, Experiment. Math. 13 (2004), $361-382$.

[8] J. Jorgenson, L. Smajlović, and H. Then, Kronecker's limit formula, holomorphic modular functions and $q$-expansions on certain arithmetic groups, Exp. Math. 25 (2016), 295-320.

[9] J. Jorgenson, L. Smajlović, and H. Then, data page http://www.efsa.unsa.ba/ lejla.smajlovic/

[10] S. Lang, Introduction to Transcendental Numbers, in: Collected Papers Volume I, 1952-1970, Springer New York 2000.

[11] S. Lang, Elliptic functions, 2nd ed., Springer Verlag New York, 1987.

[12] K. Matthews, Finding the class number $h(d)$ of binary quadratic forms of negative discriminant $d$, http://www.numbertheory.org/php/classnoneg.html

[13] F. Morain, Implementing the asymptotically fast version of the elliptic curve primality proving algorithm, Math. Comp. 76 (2007), 493-505. 
[14] N. Schapperacher, On the history of Hilbert's twelfth problem: a comedy of errors. in: Matériaux pour l'histoire des mathématiques au XXe siècle (Nice, 1996), 243-273, Sémin. Congr., 3, Soc. Math. France, Paris, 1998.

[15] G. Shimura, Introduction to the Theory of Automorphic Functions, Iwanami Publ. Company and Princeton Univ. Press, 1974.

[16] H. Söhngen, Zur komplexen Multiplikation, Math. Ann. 111 (1935), 302-328.

[17] N. Yui and D. Zagier, On the sigular values of Weber modular function, Math. Comp. 66, no. 220 (1997), $1645-1662$.

[18] D. B. Zagier, Elliptic modular forms and their applications. In K. Ranestad (ed.) The 1-2-3 of Modular Forms, Universitext, Springer, Berlin, (2008), 1-103.

\section{Appendix A. List of some polynomials $P_{m, N}$ And $Q_{m, N}$}

For all positive square-free levels $N$ such that $\Gamma_{0}(N)^{+}$has genus one, and some small primes $m$, we list here polynomials $P_{m, N}$ and $Q_{m, N}$ which we employ in Proposition 5 .

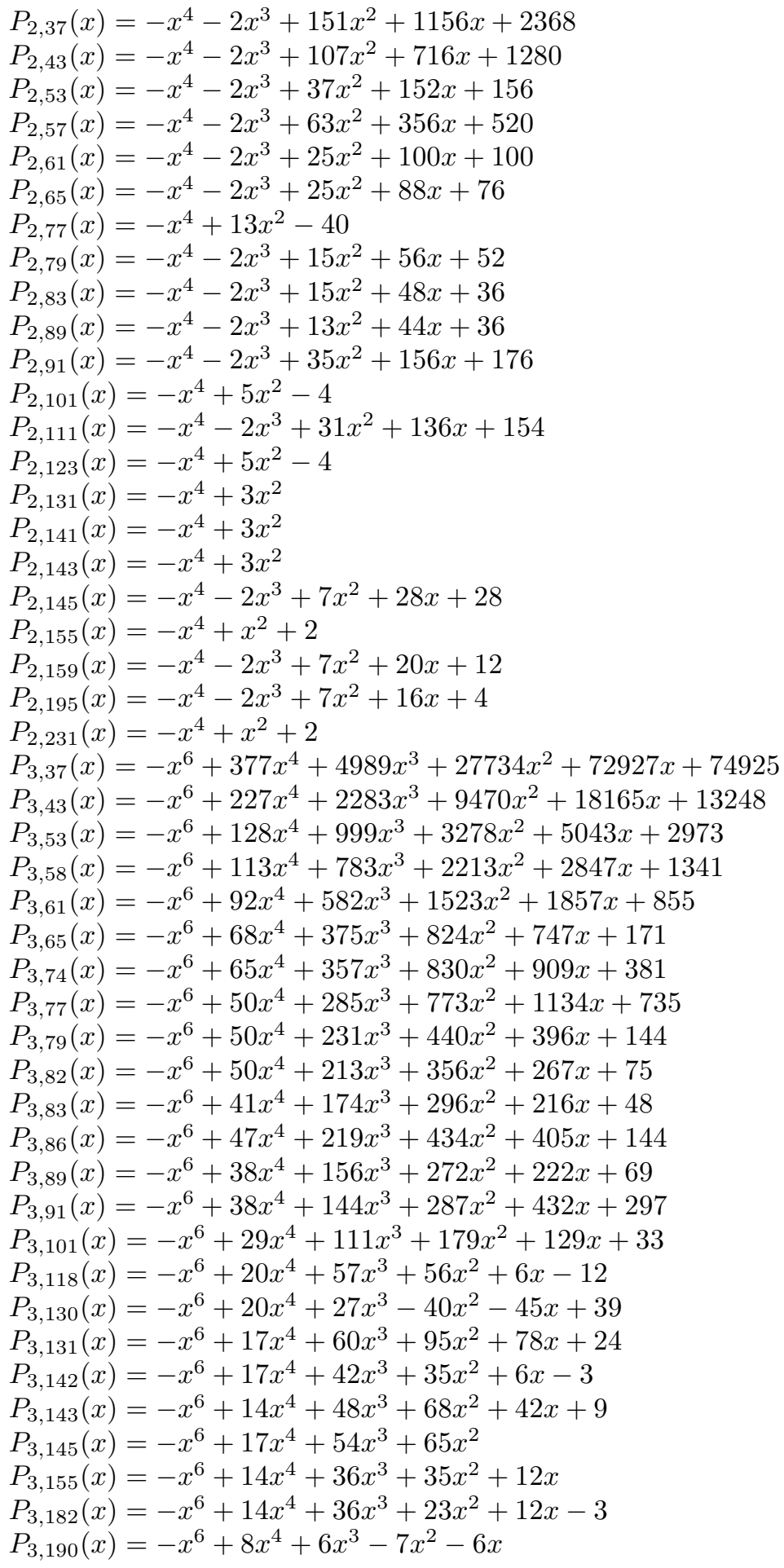

$Q_{2,37}(x)=6 x^{2}+60 x+148$ $Q_{2,43}(x)=6 x^{2}+52 x+112$ $Q_{2,53}(x)=4 x^{2}+18 x+20$ $Q_{2,57}(x)=6 x^{2}+44 x+76$ $Q_{2,61}(x)=4 x^{2}+18 x+20$ $Q_{2,65}(x)=4 x^{2}+14 x+12$ $Q_{2,77}(x)=2 x^{2}-10$ $Q_{2,79}(x)=4 x^{2}+16 x+16$ $Q_{2,83}(x)=4 x^{2}+14 x+12$ $Q_{2,89}(x)=4 x^{2}+14 x+12$ $Q_{2,91}(x)=6 x^{2}+36 x+56$ $Q_{2,101}(x)=2 x^{2}-2$ $Q_{2,111}(x)=6 x^{2}+36 x+52$ $Q_{2,123}(x)=2 x^{2}-2$ $Q_{2,131}(x)=2 x^{2}$ $Q_{2,141}(x)=2 x^{2}$ $Q_{2,143}(x)=2 x^{2}$

$Q_{2,145}(x)=4 x^{2}+14 x+12$ $Q_{2,155}(x)=2 x^{2}+2$ $Q_{2,159}(x)=4 x^{2}+12 x+8$ $Q_{2,195}(x)=4 x^{2}+14 x+12$ $Q_{2,231}(x)=2 x^{2}+2$ $Q_{3,37}(x)=6 x^{4}+108 x^{3}+732 x^{2}+2220 x+2553$ $Q_{3,43}(x)=6 x^{4}+81 x^{3}+399 x^{2}+843 x+639$ $Q_{3,53}(x)=3 x^{4}+36 x^{3}+159 x^{2}+300 x+201$ $Q_{3,58}(x)=3 x^{4}+24 x^{3}+69 x^{2}+78 x+18$ $Q_{3,61}(x)=3 x^{4}+27 x^{3}+96 x^{2}+156 x+90$ $Q_{3,65}(x)=3 x^{4}+27 x^{3}+78 x^{2}+66 x-18$ $Q_{3,74}(x)=12 x^{3}+72 x^{2}+132 x+75$ $Q_{3,77}(x)=12 x^{3}+72 x^{2}+168 x+147$ $Q_{3,79}(x)=3 x^{4}+18 x^{3}+42 x^{2}+48 x+24$ $Q_{3,82}(x)=3 x^{4}+12 x^{3}+12 x^{2}-3$ $Q_{3,83}(x)=3 x^{4}+18 x^{3}+36 x^{2}+24 x$

$Q_{3,86}(x)=9 x^{3}+51 x^{2}+87 x+45$ $Q_{3,89}(x)=3 x^{4}+15 x^{3}+30 x^{2}+27 x+9$ $Q_{3,91}(x)=6 x^{4}+33 x^{3}+66 x^{2}+111 x+99$ $Q_{3,101}(x)=9 x^{3}+33 x^{2}+36 x+12$ $Q_{3,118}(x)=3 x^{4}+9 x^{3}+3 x^{2}-18 x-18$ $Q_{3,130}(x)=-3 x^{4}+3 x^{3}+36 x^{2}+6 x-42$ $Q_{3,131}(x)=6 x^{3}+21 x^{2}+27 x+9$ $Q_{3,142}(x)=3 x^{4}+6 x^{3}-6 x-3$ $Q_{3,143}(x)=6 x^{3}+18 x^{2}+18 x+6$ $Q_{3,145}(x)=3 x^{4}+9 x^{3}+15 x^{2}$ $Q_{3,155}(x)=6 x^{3}+12 x^{2}+6 x$ $Q_{3,182}(x)=3 x^{3}+12 x^{2}+15 x-3$ $Q_{3,190}(x)=3 x^{4}+6 x^{3}-6 x-3$ 
$P_{3,238}(x)=-x^{6}-x^{4}+12 x^{3}+2 x^{2}-12 x$

$Q_{3,238}(x)=3 x^{4}+6 x^{3}+15 x^{2}-6 x-18$

$P_{5,37}(x)=-x^{10}-20 x^{9}+990 x^{8}+42370 x^{7}+703582 x^{6}+6568735 x^{5}+37836405 x^{4}+136785100 x^{3}+299887664 x^{2}+$ $358376080 x+173041600$

$Q_{5,37}(x)=10 x^{8}+545 x^{7}+11650 x^{6}+131125 x^{5}+855770 x^{4}+3288645 x^{3}+7041840 x^{2}+6967840 x+1423760$

$P_{5,43}(x)=-x^{10}-20 x^{9}+265 x^{8}+12705 x^{7}+181287 x^{6}+1411215 x^{5}+6698460 x^{4}+19703790 x^{3}+34400079 x^{2}+$

$31328665 x+10280215$

$Q_{5,43}(x)=10 x^{8}+385 x^{7}+6155 x^{6}+53205 x^{5}+268515 x^{4}+785665 x^{3}+1199105 x^{2}+606820 x-292160$ 521600

$P_{5,53}(x)=-x^{10}-5 x^{9}+365 x^{8}+6920 x^{7}+58782 x^{6}+292265 x^{5}+918570 x^{4}+1853500 x^{3}+2333784 x^{2}+1672320 x+$

$Q_{5,53}(x)=5 x^{8}+135 x^{7}+1530 x^{6}+9545 x^{5}+35960 x^{4}+84020 x^{3}+119280 x^{2}+94400 x+32000$

$P_{5,57}(x)=-x^{10}-20 x^{9}-105 x^{8}+1080 x^{7}+17847 x^{6}+102925 x^{5}+303155 x^{4}+472920 x^{3}+357079 x^{2}+92245 x-8825$

$Q_{5,57}(x)=10 x^{8}+250 x^{7}+2610 x^{6}+14030 x^{5}+40760 x^{4}+62000 x^{3}+42145 x^{2}+5170 x-4075$

245400

$P_{5,58}(x)=-x^{10}-5 x^{9}+285 x^{8}+4955 x^{7}+37577 x^{6}+160715 x^{5}+404395 x^{4}+554805 x^{3}+262984 x^{2}-229910 x-$

$Q_{5,58}(x)=5 x^{8}+100 x^{7}+680 x^{6}+770 x^{5}-13820 x^{4}-83240 x^{3}-213100 x^{2}-266750 x-133125$

$P_{5,61}(x)=-x^{10}-5 x^{9}+185 x^{8}+2830 x^{7}+18652 x^{6}+70345 x^{5}+162550 x^{4}+229740 x^{3}+187624 x^{2}+76800 x+10880$

$Q_{5,61}(x)=5 x^{8}+100 x^{7}+820 x^{6}+3530 x^{5}+8410 x^{4}+10400 x^{3}+4520 x^{2}-2240 x-1920$

$P_{5,74}(x)=-x^{10}+230 x^{8}+2690 x^{7}+15182 x^{6}+50995 x^{5}+107545 x^{4}+140020 x^{3}+102384 x^{2}+31680 x$

$Q_{5,74}(x)=35 x^{7}+560 x^{6}+3635 x^{5}+12240 x^{4}+22495 x^{3}+21240 x^{2}+7920 x$

$P_{5,77}(x)=-x^{10}+175 x^{8}+1845 x^{7}+9757 x^{6}+33170 x^{5}+82775 x^{4}+166330 x^{3}+265219 x^{2}+281930 x+138375$

$Q_{5,77}(x)=40 x^{7}+485 x^{6}+2505 x^{5}+7555 x^{4}+16795 x^{3}+32285 x^{2}+44660 x+27675$

$P_{5,79}(x)=-x^{10}-5 x^{9}+65 x^{8}+760 x^{7}+3402 x^{6}+8240 x^{5}+11540 x^{4}+9280 x^{3}+4224 x^{2}+1280 x+320$

$Q_{5,79}(x)=5 x^{8}+60 x^{7}+285 x^{6}+650 x^{5}+640 x^{4}-20 x^{3}-480 x^{2}-240 x$

$P_{5,82}(x)=-x^{10}-5 x^{9}+70 x^{8}+780 x^{7}+3127 x^{6}+5565 x^{5}+1630 x^{4}-9180 x^{3}-12826 x^{2}-5160 x$

$Q_{5,82}(x)=5 x^{8}+50 x^{7}+95 x^{6}-670 x^{5}-3855 x^{4}-8000 x^{3}-7445 x^{2}-2580 x$

$P_{5,83}(x)=-x^{10}-5 x^{9}+50 x^{8}+570 x^{7}+2472 x^{6}+6065 x^{5}+9240 x^{4}+8835 x^{3}+5024 x^{2}+1470 x+180$

$Q_{5,83}(x)=5 x^{8}+55 x^{7}+245 x^{6}+555 x^{5}+615 x^{4}+105 x^{3}-530 x^{2}-570 x-180$

$P_{5,86}(x)=-x^{10}+145 x^{8}+1365 x^{7}+6327 x^{6}+17955 x^{5}+33300 x^{4}+40730 x^{3}+31879 x^{2}+14625 x+3015$

$Q_{5,86}(x)=25 x^{7}+315 x^{6}+1645 x^{5}+4635 x^{4}+7665 x^{3}+7515 x^{2}+4100 x+960$

$P_{5,89}(x)=-x^{10}-5 x^{9}+40 x^{8}+400 x^{7}+1287 x^{6}+1725 x^{5}+310 x^{4}-1380 x^{3}-936 x^{2}$

$Q_{5,89}(x)=5 x^{8}+50 x^{7}+175 x^{6}+200 x^{5}-190 x^{4}-600 x^{3}-360 x^{2}$

$P_{5,91}(x)=-x^{10}-20 x^{9}-75 x^{8}+460 x^{7}+3952 x^{6}+9065 x^{5}+1620 x^{4}-19110 x^{3}-15476 x^{2}+10335 x+9250$

$Q_{5,91}(x)=10 x^{8}+170 x^{7}+1030 x^{6}+2510 x^{5}+910 x^{4}-5310 x^{3}-5265 x^{2}+2820 x+3125$

$P_{5,101}(x)=-x^{10}+85 x^{8}+615 x^{7}+2162 x^{6}+4515 x^{5}+5860 x^{4}+4650 x^{3}+2114 x^{2}+480 x+40$

$Q_{5,101}(x)=20 x^{7}+185 x^{6}+685 x^{5}+1300 x^{4}+1345 x^{3}+745 x^{2}+200 x+20$

$P_{5,102}(x)=-x^{10}-5 x^{9}+25 x^{8}+295 x^{7}+912 x^{6}+645 x^{5}-1955 x^{4}-4135 x^{3}-2521 x^{2}-40 x+300$

$Q_{5,102}(x)=5 x^{8}+40 x^{7}+50 x^{6}-510 x^{5}-1995 x^{4}-2700 x^{3}-1400 x^{2}-70 x+100$

$P_{5,111}(x)=-x^{10}-20 x^{9}-30 x^{8}+985 x^{7}+4057 x^{6}-8645 x^{5}-66360 x^{4}-43610 x^{3}+285224 x^{2}+568780 x+295600$

$Q_{5,111}(x)=10 x^{8}+155 x^{7}+580 x^{6}-1775 x^{5}-13915 x^{4}-10920 x^{3}+68610 x^{2}+149200 x+81500$

$P_{5,114}(x)=-x^{10}+75 x^{8}+480 x^{7}+1527 x^{6}+3025 x^{5}+3415 x^{4}+1050 x^{3}-1501 x^{2}-1365 x-225$

$Q_{5,114}(x)=10 x^{7}+130 x^{6}+630 x^{5}+1390 x^{4}+1240 x^{3}-145 x^{2}-870 x-225$

$P_{5,118}(x)=-x^{10}-5 x^{9}+20 x^{8}+185 x^{7}+477 x^{6}+520 x^{5}+175 x^{4}-80 x^{3}-51 x^{2}$

$Q_{5,118}(x)=5 x^{8}+30 x^{7}+20 x^{6}-230 x^{5}-660 x^{4}-690 x^{3}-255 x^{2}$

$P_{5,123}(x)=-x^{10}+50 x^{8}+280 x^{7}+772 x^{6}+1275 x^{5}+1315 x^{4}+840 x^{3}+304 x^{2}+35 x-10$

$Q_{5,123}(x)=15 x^{7}+100 x^{6}+270 x^{5}+390 x^{4}+330 x^{3}+150 x^{2}+15 x-10$

$P_{5,131}(x)=-x^{10}+40 x^{8}+210 x^{7}+562 x^{6}+920 x^{5}+970 x^{4}+635 x^{3}+234 x^{2}+30 x$

$Q_{5,131}(x)=15 x^{7}+80 x^{6}+180 x^{5}+220 x^{4}+140 x^{3}+40 x^{2}$

$P_{5,138}(x)=-x^{10}-5 x^{9}+5 x^{8}+95 x^{7}+187 x^{6}-295 x^{5}-925 x^{4}+325 x^{3}+1274 x^{2}-120 x-540$

$Q_{5,138}(x)=5 x^{8}+30 x^{7}+5 x^{6}-310 x^{5}-525 x^{4}+490 x^{3}+1055 x^{2}-210 x-540$

$P_{5,141}(x)=-x^{10}+40 x^{8}+190 x^{7}+432 x^{6}+560 x^{5}+375 x^{4}+60 x^{3}-36 x^{2}$

$Q_{5,141}(x)=10 x^{7}+70 x^{6}+180 x^{5}+200 x^{4}+80 x^{3}$

$P_{5,142}(x)=-x^{10}-5 x^{9}+15 x^{8}+120 x^{7}+142 x^{6}-280 x^{5}-580 x^{4}+120 x^{3}+619 x^{2}+45 x-195$

$Q_{5,142}(x)=5 x^{8}+25 x^{7}-10 x^{6}-215 x^{5}-240 x^{4}+245 x^{3}+360 x^{2}-75 x-95$

$P_{5,143}(x)=-x^{10}+35 x^{8}+190 x^{7}+537 x^{6}+890 x^{5}+850 x^{4}+420 x^{3}+49 x^{2}-30 x$

$Q_{5,143}(x)=10 x^{7}+65 x^{6}+205 x^{5}+375 x^{4}+340 x^{3}+85 x^{2}-30 x$

$P_{5,159}(x)=-x^{10}-5 x^{9}+5 x^{8}+50 x^{7}+42 x^{6}-10 x^{5}+90 x^{4}+115 x^{3}-96 x^{2}-150 x-40$ 
$Q_{5,159}(x)=5 x^{8}+30 x^{7}+45 x^{6}+5 x^{5}+35 x^{4}+95 x^{3}-45 x^{2}-130 x-40$

$P_{5,174}(x)=-x^{10}-5 x^{9}+50 x^{7}+137 x^{6}+245 x^{5}+100 x^{4}-210 x^{3}-236 x^{2}-80 x$

$Q_{5,174}(x)=5 x^{8}+25 x^{7}+35 x^{6}-55 x^{5}-260 x^{4}-650 x^{3}-460 x^{2}-80 x$

$P_{5,182}(x)=-x^{10}+25 x^{8}+80 x^{7}+132 x^{6}+105 x^{5}-20 x^{4}-120 x^{3}-96 x^{2}-15 x+10$

$Q_{5,182}(x)=10 x^{7}+30 x^{6}-10 x^{5}-110 x^{4}-150 x^{3}-95 x^{2}-20 x+5$

$P_{5,222}(x)=-x^{10}+20 x^{8}+65 x^{7}+77 x^{6}-5 x^{5}-80 x^{4}-50 x^{3}+24 x^{2}$

$Q_{5,222}(x)=5 x^{7}+20 x^{6}+35 x^{5}+15 x^{4}-20 x^{3}-30 x^{2}$

$P_{5,231}(x)=-x^{10}+10 x^{8}+30 x^{7}+37 x^{6}-10 x^{5}-40 x^{4}-110 x^{3}+64 x^{2}+20 x$

$Q_{5,231}(x)=10 x^{7}+20 x^{6}-80 x^{4}-50 x^{3}+80 x^{2}+20 x$

$P_{5,238}(x)=-x^{10}-5 x^{9}-5 x^{8}-15 x^{7}+32 x^{6}+160 x^{5}+270 x^{4}+500 x^{3}+704 x^{2}+480 x+120$

$Q_{5,238}(x)=5 x^{8}+20 x^{7}+40 x^{6}+80 x^{5}+15 x^{4}-240 x^{3}-260 x^{2}+60$

$P_{7,37}(x)=-x^{14}-56 x^{13}+4683 x^{12}+356538 x^{11}+10437455 x^{10}+179095609 x^{9}+2043824848 x^{8}+16416622348 x^{7}+$ $95299084468 x^{6}+402982198983 x^{5}+1231845056484 x^{4}+2653820294895 x^{3}+3822937817418 x^{2}+3304459709523 x+$ 1295301173229

$Q_{7,37}(x)=14 x^{12}+1806 x^{11}+75208 x^{10}+1652462 x^{9}+22684179 x^{8}+210411278 x^{7}+1370543307 x^{6}+6363965517 x^{5}+$ $20996395378 x^{4}+48110934795 x^{3}+72744325857 x^{2}+65162885886 x+26129994849$

$P_{7,43}(x)=-x^{14}-56 x^{13}+182 x^{12}+60970 x^{11}+1695491 x^{10}+24797409 x^{9}+231126919 x^{8}+1468533199 x^{7}+$ $6513250870 x^{6}+20085965795 x^{5}+41623467914 x^{4}+52910061015 x^{3}+30627922338 x^{2}-7483942620 x-14454403425$ $Q_{7,43}(x)=14 x^{12}+1197 x^{11}+36974 x^{10}+605955 x^{9}+6033503 x^{8}+38254538 x^{7}+151350381 x^{6}+314848828 x^{5}-$ $60459406 x^{4}-2319854579 x^{3}-6405785505 x^{2}-8075095875 x-4113041625$

$P_{7,53}(x)=-x^{14}-14 x^{13}+1162 x^{12}+40005 x^{11}+606536 x^{10}+5613370 x^{9}+35390329 x^{8}+160064135 x^{7}+$ $532591129 x^{6}+1314475750 x^{5}+2390484292 x^{4}+3126753154 x^{3}+2794167340 x^{2}+1532423424 x+390166077$

$Q_{7,53}(x)=7 x^{12}+392 x^{11}+8673 x^{10}+107037 x^{9}+847987 x^{8}+4636772 x^{7}+18193154 x^{6}+52099663 x^{5}+$ $108724896 x^{4}+161792981 x^{3}+163201423 x^{2}+100222367 x+28331632$

$P_{7,57}(x)=-x^{14}-56 x^{13}-1211 x^{12}-11830 x^{11}-28014 x^{10}+501949 x^{9}+5593555 x^{8}+26829292 x^{7}+65375912 x^{6}+$ $45090304 x^{5}-187430922 x^{4}-593974906 x^{3}-769009137 x^{2}-486751265 x-122816694$

$Q_{7,57}(x)=14 x^{12}+714 x^{11}+15050 x^{10}+171514 x^{9}+1158780 x^{8}+4712330 x^{7}+10542567 x^{6}+5975256 x^{5}-$ $33215945 x^{4}-99806728 x^{3}-127840713 x^{2}-80862782 x-20469449$

$P_{7,58}(x)=-x^{14}-14 x^{13}+854 x^{12}+29988 x^{11}+446068 x^{10}+3960922 x^{9}+23509229 x^{8}+98748860 x^{7}+303624587 x^{6}+$ $696533866 x^{5}+1197037604 x^{4}+1514923312 x^{3}+1342478626 x^{2}+744983610 x+194492025$

$Q_{7,58}(x)=7 x^{12}+273 x^{11}+3836 x^{10}+23163 x^{9}+21651 x^{8}-529970 x^{7}-3422356 x^{6}-9904006 x^{5}-13846791 x^{4}-$ $2891679 x^{3}+18881128 x^{2}+25322955 x+10727325$

$P_{7,61}(x)=-x^{14}-14 x^{13}+448 x^{12}+13909 x^{11}+172361 x^{10}+1268722 x^{9}+6238500 x^{8}+21599081 x^{7}+53953634 x^{6}+$ $97890737 x^{5}+127912869 x^{4}+117275718 x^{3}+71531802 x^{2}+26028135 x+4266675$

$Q_{7,61}(x)=7 x^{12}+266 x^{11}+4151 x^{10}+36575 x^{9}+206752 x^{8}+799169 x^{7}+2181564 x^{6}+4256770 x^{5}+5908798 x^{4}+$ $5699323 x^{3}+3628611 x^{2}+1369305 x+231525$

$P_{7,65}(x)=-x^{14}-14 x^{13}+315 x^{12}+10171 x^{11}+125986 x^{10}+927906 x^{9}+4545011 x^{8}+15372700 x^{7}+35691999 x^{6}+$ $53226236 x^{5}+38728333 x^{4}-16515191 x^{3}-63330744 x^{2}-53021808 x-15760899$

$Q_{7,65}(x)=7 x^{12}+217 x^{11}+2919 x^{10}+24101 x^{9}+139832 x^{8}+595658 x^{7}+1826244 x^{6}+3732386 x^{5}+4219509 x^{4}+$ $630749 x^{3}-4483731 x^{2}-4983111 x-1704780$

$P_{7,74}(x)=-x^{14}+707 x^{12}+13930 x^{11}+131943 x^{10}+758499 x^{9}+2893564 x^{8}+7649446 x^{7}+14294658 x^{6}+$ $18929043 x^{5}+17469970 x^{4}+10742137 x^{3}+3980542 x^{2}+688485 x+10157$

$Q_{7,74}(x)=98 x^{11}+2688 x^{10}+29540 x^{9}+175903 x^{8}+641102 x^{7}+1520295 x^{6}+2412977 x^{5}+2564170 x^{4}+1761809 x^{3}+$ $711249 x^{2}+130718 x+1939$

$P_{7,79}(x)=-x^{14}-14 x^{13}+21 x^{12}+1743 x^{11}+16506 x^{10}+80759 x^{9}+233970 x^{8}+385196 x^{7}+215544 x^{6}-475524 x^{5}-$ $1233960 x^{4}-1332464 x^{3}-787424 x^{2}-247296 x-32256$

$Q_{7,79}(x)=7 x^{12}+154 x^{11}+1407 x^{10}+6650 x^{9}+14994 x^{8}-2800 x^{7}-116116 x^{6}-345016 x^{5}-538104 x^{4}-500864 x^{3}-$ $277536 x^{2}-84224 x-10752$

$P_{7,82}(x)=-x^{14}-14 x^{13}+77 x^{12}+3066 x^{11}+30240 x^{10}+162778 x^{9}+541753 x^{8}+1117291 x^{7}+1214136 x^{6}-$ $140910 x^{5}-2638146 x^{4}-4157811 x^{3}-3307683 x^{2}-1387400 x-243376$

$Q_{7,82}(x)=7 x^{12}+126 x^{11}+651 x^{10}-2296 x^{9}-46564 x^{8}-278040 x^{7}-960295 x^{6}-2163672 x^{5}-3290553 x^{4}-$ $3358782 x^{3}-2205350 x^{2}-840336 x-140896$

$P_{7,83}(x)=-x^{14}-14 x^{13}-14 x^{12}+1106 x^{11}+11844 x^{10}+65170 x^{9}+227901 x^{8}+535283 x^{7}+840105 x^{6}+811363 x^{5}+$ $310807 x^{4}-281302 x^{3}-463744 x^{2}-260232 x-55440$

$Q_{7,83}(x)=7 x^{12}+140 x^{11}+1225 x^{10}+5978 x^{9}+16597 x^{8}+18928 x^{7}-37128 x^{6}-202930 x^{5}-424403 x^{4}-523362 x^{3}-$ $396452 x^{2}-171192 x-32256$ 
$P_{7,86}(x)=-x^{14}+406 x^{12}+6314 x^{11}+48195 x^{10}+227353 x^{9}+724355 x^{8}+1630615 x^{7}+2658026 x^{6}+3173079 x^{5}+$ $2772798 x^{4}+1747599 x^{3}+762726 x^{2}+208320 x+26775$

$Q_{7,86}(x)=63 x^{11}+1344 x^{10}+11963 x^{9}+59325 x^{8}+184660 x^{7}+384839 x^{6}+556920 x^{5}+568624 x^{4}+408653 x^{3}+$ $200767 x^{2}+61565 x+8925$

$P_{7,89}(x)=-x^{14}-14 x^{13}-42 x^{12}+497 x^{11}+5803 x^{10}+29218 x^{9}+85962 x^{8}+147665 x^{7}+101430 x^{6}-148848 x^{5}-$ $476154 x^{4}-586355 x^{3}-397902 x^{2}-145320 x-22239$

$Q_{7,89}(x)=7 x^{12}+126 x^{11}+973 x^{10}+4025 x^{9}+8316 x^{8}-483 x^{7}-53760 x^{6}-163044 x^{5}-267120 x^{4}-270900 x^{3}-$ $169344 x^{2}-59724 x-9072$

$P_{7,101}(x)=-x^{14}+210 x^{12}+2387 x^{11}+13461 x^{10}+47572 x^{9}+115747 x^{8}+204029 x^{7}+267806 x^{6}+264229 x^{5}+$ $194439 x^{4}+103712 x^{3}+37638 x^{2}+8071 x+700$

$Q_{7,101}(x)=49 x^{11}+686 x^{10}+4116 x^{9}+14280 x^{8}+32571 x^{7}+52164 x^{6}+60557 x^{5}+51184 x^{4}+30828 x^{3}+12474 x^{2}+$ $2919 x+252$

$P_{7,102}(x)=-x^{14}-14 x^{13}+14 x^{12}+1211 x^{11}+8351 x^{10}+25480 x^{9}+32468 x^{8}-27349 x^{7}-174951 x^{6}-251048 x^{5}-$ $63567 x^{4}+178486 x^{3}+208137 x^{2}+62258 x+525$

$Q_{7,102}(x)=7 x^{12}+91 x^{11}+210 x^{10}-2086 x^{9}-15673 x^{8}-48916 x^{7}-85113 x^{6}-67228 x^{5}+26726 x^{4}+85351 x^{3}+$ $78309 x^{2}+28084 x+238$

$P_{7,111}(x)=-x^{14}-56 x^{13}-693 x^{12}-1680 x^{11}+21287 x^{10}+157024 x^{9}+241537 x^{8}-1211084 x^{7}-5491598 x^{6}-$ $4225620 x^{5}+21857724 x^{4}+67572288 x^{3}+83377944 x^{2}+47618928 x+9525600$

$Q_{7,111}(x)=14 x^{12}+462 x^{11}+5446 x^{10}+27440 x^{9}+30618 x^{8}-276766 x^{7}-1170750 x^{6}-798504 x^{5}+5595856 x^{4}+$ $17410512 x^{3}+22169448 x^{2}+13060656 x+2667168$

$P_{7,114}(x)=-x^{14}+161 x^{12}+1330 x^{11}+4522 x^{10}+7329 x^{9}+5063 x^{8}-2926 x^{7}-18956 x^{6}-27650 x^{5}+3066 x^{4}+$ $29232 x^{3}+6579 x^{2}-8883 x+1134$

$Q_{7,114}(x)=42 x^{11}+378 x^{10}+658 x^{9}-3052 x^{8}-12306 x^{7}-12145 x^{6}+1792 x^{5}+11277 x^{4}+16884 x^{3}+63 x^{2}-$ $4158 x+567$

$P_{7,118}(x)=-x^{14}-14 x^{13}-21 x^{12}+525 x^{11}+3836 x^{10}+12964 x^{9}+26490 x^{8}+35266 x^{7}+29659 x^{6}+12194 x^{5}-$ $1701 x^{4}-3591 x^{3}-918 x^{2}$

$Q_{7,118}(x)=7 x^{12}+77 x^{11}+196 x^{10}-966 x^{9}-8036 x^{8}-26068 x^{7}-51002 x^{6}-66234 x^{5}-56595 x^{4}-28665 x^{3}-6426 x^{2}$

$P_{7,123}(x)=-x^{14}+105 x^{12}+924 x^{11}+3948 x^{10}+10094 x^{9}+16389 x^{8}+15610 x^{7}+3332 x^{6}-12992 x^{5}-19691 x^{4}-$ $13615 x^{3}-4131 x^{2}-56 x+84$

$Q_{7,123}(x)=35 x^{11}+315 x^{10}+1316 x^{9}+3318 x^{8}+5068 x^{7}+3640 x^{6}-1323 x^{5}-5285 x^{4}-4865 x^{3}-1967 x^{2}-224 x-28$

$P_{7,130}(x)=-x^{14}-14 x^{13}+91 x^{12}+2555 x^{11}+18074 x^{10}+59374 x^{9}+74083 x^{8}-104020 x^{7}-488369 x^{6}-599396 x^{5}-$ $37023 x^{4}+626241 x^{3}+562064 x^{2}+27804 x-141463$

$Q_{7,130}(x)=-7 x^{12}-35 x^{11}+847 x^{10}+10577 x^{9}+50442 x^{8}+108766 x^{7}+25816 x^{6}-391342 x^{5}-830697 x^{4}-$ $536907 x^{3}+388521 x^{2}+798189 x+375830$

$P_{7,131}(x)=-x^{14}+91 x^{12}+735 x^{11}+3108 x^{10}+8666 x^{9}+17341 x^{8}+25788 x^{7}+28721 x^{6}+23625 x^{5}+13762 x^{4}+$ $5180 x^{3}+1000 x^{2}$

$Q_{7,131}(x)=28 x^{11}+259 x^{10}+1099 x^{9}+2877 x^{8}+5173 x^{7}+6615 x^{6}+5999 x^{5}+3710 x^{4}+1428 x^{3}+280 x^{2}$

$P_{7,138}(x)=-x^{14}-14 x^{13}+518 x^{11}+1701 x^{10}+833 x^{9}-1916 x^{8}-588 x^{7}+1757 x^{6}+224 x^{5}-3976 x^{4}-3850 x^{3}+$ $1175 x^{2}+2877 x+1260$

$Q_{7,138}(x)=7 x^{12}+63 x^{11}+7 x^{10}-1106 x^{9}-2261 x^{8}+343 x^{7}+3766 x^{6}+3199 x^{5}-833 x^{4}-2982 x^{3}-1001 x^{2}+483 x+315$

$P_{7,141}(x)=-x^{14}+70 x^{12}+497 x^{11}+1750 x^{10}+3829 x^{9}+5805 x^{8}+6461 x^{7}+5600 x^{6}+4088 x^{5}+2793 x^{4}+1778 x^{3}+$ $951 x^{2}+336 x+63$

$Q_{7,141}(x)=28 x^{11}+182 x^{10}+574 x^{9}+1183 x^{8}+1750 x^{7}+2037 x^{6}+1932 x^{5}+1575 x^{4}+1064 x^{3}+574 x^{2}+210 x+42$

$P_{7,142}(x)=-x^{14}-14 x^{13}-42 x^{12}+224 x^{11}+1701 x^{10}+3290 x^{9}-2028 x^{8}-15792 x^{7}-16471 x^{6}+9926 x^{5}+$ $29414 x^{4}+13328 x^{3}-9549 x^{2}-10962 x-3024$

$Q_{7,142}(x)=7 x^{12}+70 x^{11}+147 x^{10}-826 x^{9}-4998 x^{8}-9576 x^{7}-3262 x^{6}+14168 x^{5}+19383 x^{4}+3346 x^{3}-$ $9765 x^{2}-7182 x-1512$ $484 x^{2}$

$P_{7,143}(x)=-x^{14}+77 x^{12}+567 x^{11}+2100 x^{10}+4851 x^{9}+7688 x^{8}+9009 x^{7}+7973 x^{6}+4445 x^{5}+287 x^{4}-1232 x^{3}-$

$Q_{7,143}(x)=21 x^{11}+189 x^{10}+770 x^{9}+1918 x^{8}+3241 x^{7}+3689 x^{6}+2520 x^{5}+756 x^{4}$

$P_{7,145}(x)=-x^{14}-14 x^{13}-42 x^{12}+161 x^{11}+1001 x^{10}+602 x^{9}-4632 x^{8}-6881 x^{7}+7378 x^{6}+17248 x^{5}-2625 x^{4}-$ $16975 x^{3}-4460 x^{2}+2100 x$

$Q_{7,145}(x)=7 x^{12}+77 x^{11}+252 x^{10}-63 x^{9}-1757 x^{8}-1995 x^{7}+3360 x^{6}+6216 x^{5}-1715 x^{4}-6930 x^{3}-2170 x^{2}+1050 x$

$P_{7,155}(x)=-x^{14}+56 x^{12}+371 x^{11}+1295 x^{10}+2688 x^{9}+2585 x^{8}-1379 x^{7}-6041 x^{6}-4634 x^{5}+1281 x^{4}+3388 x^{3}+$ $1007 x^{2}-434 x-182$

$Q_{7,155}(x)=21 x^{11}+140 x^{10}+462 x^{9}+896 x^{8}+483 x^{7}-1533 x^{6}-2513 x^{5}-63 x^{4}+2016 x^{3}+833 x^{2}-469 x-273$ 


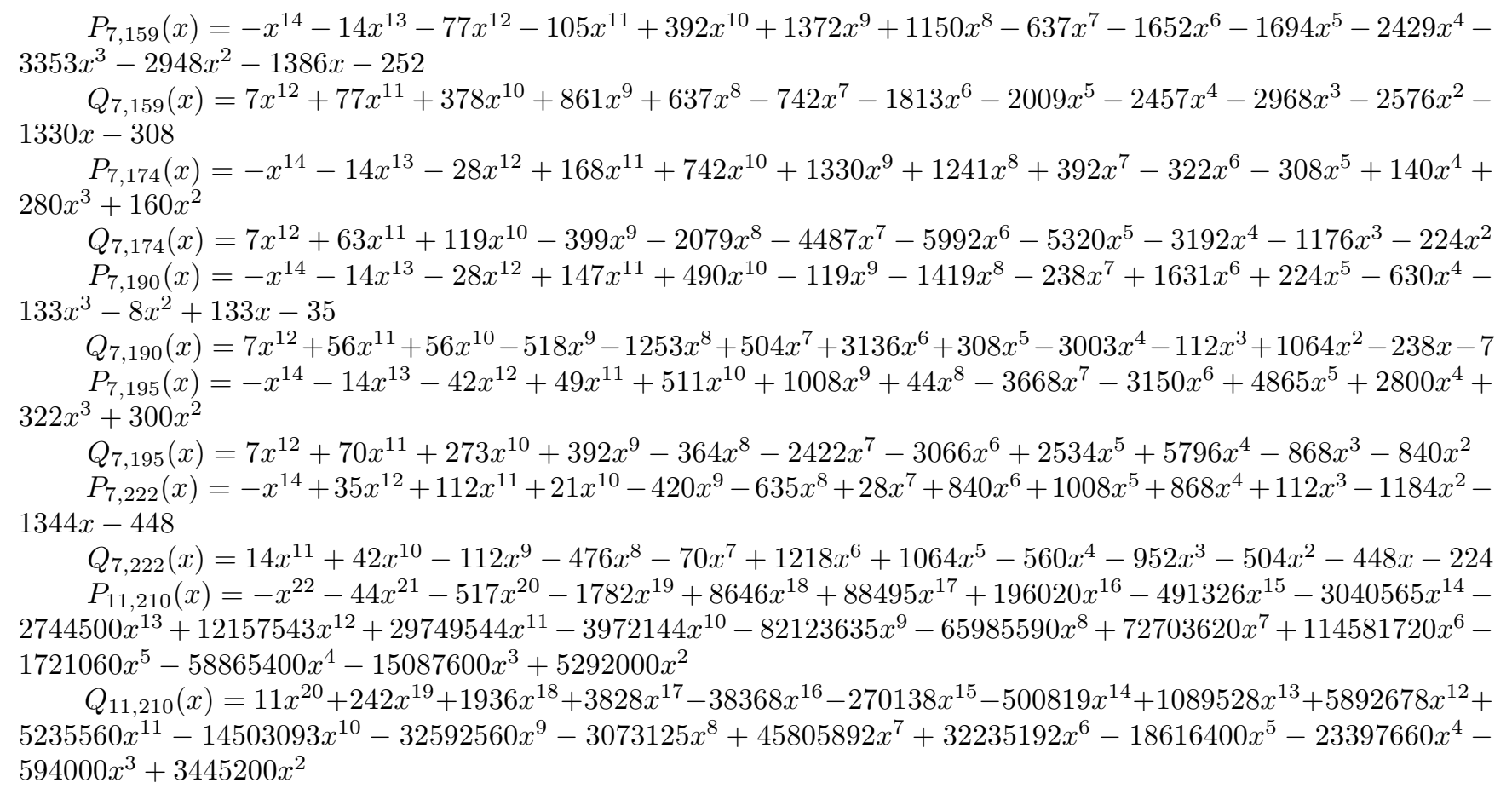

Appendix B. List of generating polynomials for $x_{N}(z)$ AT the CM POint $z=i \sqrt{m / N}$

For all positive square-free levels $N$ such that $\Gamma_{0}(N)^{+}$has genus one, and some small primes $m$, we list here the generating polynomial (17) of $x_{N}$ at the CM point $z=i \sqrt{m / N}$.

$m=2$ :

$$
\begin{array}{ll}
N=37: & x^{8}+4 x^{7}-556 x^{6}-11724 x^{5}-110853 x^{4}-588596 x^{3}-1818476 x^{2}-3066560 x-2190400=0 \\
N=43: & x^{8}+4 x^{7}-396 x^{6}-7300 x^{5}-59945 x^{4}-276524 x^{3}-743600 x^{2}-1094208 x-684032=0 \\
N=53: & x^{8}-168 x^{6}-1644 x^{5}-7533 x^{4}-19608 x^{3}-29848 x^{2}-24864 x-8784=0 \\
N=57: & x^{8}+4 x^{7}-236 x^{6}-3420 x^{5}-21125 x^{4}-71140 x^{3}-136084 x^{2}-139136 x-59136=0 \\
N=61: & x^{8}-128 x^{6}-1068 x^{5}-4101 x^{4}-8792 x^{3}-10840 x^{2}-7200 x-2000=0 \\
N=65: & x^{8}-108 x^{6}-916 x^{5}-3737 x^{4}-8728 x^{3}-11832 x^{2}-8608 x-2576=0 \\
N=77: & x^{8}-4 x^{7}-44 x^{6}-16 x^{5}+341 x^{4}+460 x^{3}-610 x^{2}-1400 x-600=0 \\
N=79: & x^{8}-82 x^{6}-528 x^{5}-1511 x^{4}-2304 x^{3}-1896 x^{2}-768 x-112=0 \\
N=83: & x^{8}-72 x^{6}-476 x^{5}-1501 x^{4}-2728 x^{3}-2928 x^{2}-1728 x-432=0 \\
N=89: & x^{8}-68 x^{6}-404 x^{5}-1089 x^{4}-1624 x^{3}-1400 x^{2}-672 x-144=0 \\
N=91: & x^{8}+4 x^{7}-108 x^{6}-1268 x^{5}-6233 x^{4}-16732 x^{3}-25128 x^{2}-18976 x-4864=0 \\
N=101: & x^{8}-4 x^{7}-20 x^{6}-8 x^{5}+37 x^{4}+28 x^{3}-18 x^{2}-16 x=0 \\
N=111: & x^{8}+4 x^{7}-100 x^{6}-972 x^{5}-3129 x^{4}-1820 x^{3}+11764 x^{2}+26680 x+17372=0 \\
N=123: & x^{8}-4 x^{7}-12 x^{6}+21 x^{4}+12 x^{3}-10 x^{2}-8 x=0 \\
N=131: & x^{8}-4 x^{7}-8 x^{6}-8 x^{5}-5 x^{4}=0 \\
N=141: & x^{8}-4 x^{7}-8 x^{6}+3 x^{4}=0 \\
N=143: & x^{8}-4 x^{7}-8 x^{6}+11 x^{4}=0 \\
N=145: & x^{8}-40 x^{6}-140 x^{5}-117 x^{4}+120 x^{3}+160 x^{2}+16=0 \\
N=155: & x^{8}-4 x^{7}-4 x^{6}-8 x^{5}-11 x^{4}-4 x^{3}-6 x^{2}=0 \\
N=159: & x^{8}-30 x^{6}-120 x^{5}-243 x^{4}-336 x^{3}-328 x^{2}-192 x-48=0 \\
N=195: & x^{8}-24 x^{6}-76 x^{5}-77 x^{4}+56 x^{3}+192 x^{2}+128 x+16=0 \\
N=231: & x^{8}-4 x^{7}+4 x^{6}-16 x^{5}+5 x^{4}-20 x^{3}+2 x^{2}-8 x=0
\end{array}
$$

$m=3:$

$N=37: \quad x^{12}-1372 x^{10}-41190 x^{9}-619785 x^{8}-5825280 x^{7}-36892492 x^{6}-161860422 x^{5}-491879369 x^{4}-$ $1009024188 x^{3}-1314153753 x^{2}-952782930 x-273526200=0$ 
$N=43: \quad x^{12}-886 x^{10}-21360 x^{9}-258084 x^{8}-1948716 x^{7}-9926938 x^{6}-35117280 x^{5}-86430245 x^{4}-$ $144741444 x^{3}-156188520 x^{2}-96674688 x-25453440=0$

$N=53: \quad x^{12}-370 x^{10}-5634 x^{9}-41841 x^{8}-185322 x^{7}-505741 x^{6}-765534 x^{5}-205040 x^{4}+1584024 x^{3}+$ $3237198 x^{2}+2809872 x+974403=0$

$N=58: \quad x^{12}-313 x^{10}-3936 x^{9}-19908 x^{8}-22962 x^{7}+258227 x^{6}+1621248 x^{5}+4796044 x^{4}+8482950 x^{3}+$ $9163638 x^{2}+5604012 x+1492263=0$

$N=61: \quad x^{12}-265 x^{10}-3270 x^{9}-18900 x^{8}-61254 x^{7}-107344 x^{6}-52488 x^{5}+183154 x^{4}+444468 x^{3}+$ $457785 x^{2}+237600 x+50625=0$

$N=65: \quad x^{12}-217 x^{10}-2640 x^{9}-16293 x^{8}-62850 x^{7}-158692 x^{6}-250932 x^{5}-193601 x^{4}+68238 x^{3}+$ $296793 x^{2}+248184 x+72009=0$

$N=74: \quad x^{12}-130 x^{10}-822 x^{9}+189 x^{8}+22344 x^{7}+117416 x^{6}+326202 x^{5}+568015 x^{4}+643788 x^{3}+465285 x^{2}+$ $196182 x+36936=0$

$N=77: \quad x^{12}-100 x^{10}-678 x^{9}-1422 x^{8}+3336 x^{7}+27788 x^{6}+77406 x^{5}+117943 x^{4}+98784 x^{3}+36015 x^{2}=0$

$N=79: \quad x^{12}-148 x^{10}-1362 x^{9}-5832 x^{8}-13722 x^{7}-16117 x^{6}+192 x^{5}+29428 x^{4}+43872 x^{3}+31440 x^{2}+$ $11520 x+1728=0$

$N=82: \quad x^{12}-145 x^{10}-1218 x^{9}-4053 x^{8}-2904 x^{7}+23057 x^{6}+91686 x^{5}+171532 x^{4}+190212 x^{3}+127560 x^{2}+$ $47952 x+7776=0$

$N=83: \quad x^{12}-130 x^{10}-1176 x^{9}-5187 x^{8}-13584 x^{7}-21556 x^{6}-17616 x^{5}+1984 x^{4}+21504 x^{3}+23232 x^{2}+$ $11520 x+2304=0$

$N=86: \quad x^{12}-94 x^{10}-492 x^{9}+252 x^{8}+10524 x^{7}+44558 x^{6}+98940 x^{5}+133555 x^{4}+111780 x^{3}+55440 x^{2}+$ $14256 x+1296=0$

$N=89: \quad x^{12}-115 x^{10}-966 x^{9}-3921 x^{8}-9330 x^{7}-13096 x^{6}-8466 x^{5}+4057 x^{4}+13506 x^{3}+12147 x^{2}+$ $5256 x+927=0$

$N=91: \quad x^{12}-172 x^{10}-1752 x^{9}-8994 x^{8}-30312 x^{7}-75127 x^{6}-141696 x^{5}-201701 x^{4}-213582 x^{3}-156915 x^{2}-$ $58806 x=0$

$N=101: \quad x^{12}-58 x^{10}-276 x^{9}-336 x^{8}+1068 x^{7}+5051 x^{6}+10050 x^{5}+12247 x^{4}+9750 x^{3}+4956 x^{2}+1458 x+189=$

0

$N=118: \quad x^{12}-70 x^{10}-438 x^{9}-1176 x^{8}-1116 x^{7}+2141 x^{6}+8676 x^{5}+13354 x^{4}+11112 x^{3}+4560 x^{2}+288 x-288=$

0

$N=130: \quad x^{12}-49 x^{10}-108 x^{9}+543 x^{8}+2178 x^{7}+680 x^{6}-6552 x^{5}-7037 x^{4}+6462 x^{3}+10641 x^{2}-1980 x-4779=0$

$N=131: \quad x^{12}-34 x^{10}-138 x^{9}-162 x^{8}+318 x^{7}+1418 x^{6}+2316 x^{5}+2074 x^{4}+1014 x^{3}+222 x^{2}+18 x+9=0$

$N=142: \quad x^{12}-55 x^{10}-288 x^{9}-612 x^{8}-348 x^{7}+1106 x^{6}+2892 x^{5}+3313 x^{4}+2172 x^{3}+837 x^{2}+180 x+18=0$

$N=143: \quad x^{12}-28 x^{10}-114 x^{9}-174 x^{8}+90 x^{7}+968 x^{6}+2232 x^{5}+3040 x^{4}+2742 x^{3}+1638 x^{2}+594 x+99=0$

$N=145: \quad x^{12}-49 x^{10}-288 x^{9}-786 x^{8}-1044 x^{7}-229 x^{6}+1170 x^{5}+1225 x^{4}=0$

$N=155: \quad x^{12}-28 x^{10}-90 x^{9}-54 x^{8}+246 x^{7}+620 x^{6}+642 x^{5}+325 x^{4}+66 x^{3}=0$

$N=182: \quad x^{12}-28 x^{10}-72 x^{9}+114 x^{8}+660 x^{7}+713 x^{6}-384 x^{5}-905 x^{4}-258 x^{3}+177 x^{2}-18 x=0$

$N=190: \quad x^{12}-37 x^{10}-162 x^{9}-294 x^{8}-144 x^{7}+374 x^{6}+684 x^{5}+277 x^{4}-288 x^{3}-321 x^{2}-90 x=0$

$N=238: \quad x^{12}-13 x^{10}-108 x^{9}-327 x^{8}-648 x^{7}-571 x^{6}+324 x^{5}+1522 x^{4}+1728 x^{3}+36 x^{2}-1296 x-648=0$

$m=5:$

$N=37: \quad x^{20}-8200 x^{18}-608420 x^{17}-23135014 x^{16}-569497400 x^{15}-9947185220 x^{14}-129301825700 x^{13}-$ $1284872136099 x^{12}-9897138882560 x^{11}-59304722938300 x^{10}-274285679138640 x^{9}-954427348113664 x^{8}-2336811561726080 x^{7}-$ $3176045941955840 x^{6}+1802296671068160 x^{5}+20737419344331776 x^{4}+52460432342487040 x^{3}+73617646015938560 x^{2}+$ $58246692878745600 x+20450365046784000=0$

$N=43: \quad x^{20}-4390 x^{18}-247300 x^{17}-7285854 x^{16}-141388800 x^{15}-1976408330 x^{14}-20841596830 x^{13}-$ $170196959744 x^{12}-1091828865330 x^{11}-5532820203455 x^{10}-22088131666840 x^{9}-68580167678404 x^{8}-160721815151090 x^{7}-$ $265068472352465 x^{6}-244359132112280 x^{5}+70083037677776 x^{4}+606000879021620 x^{3}+924348729007040 x^{2}+693929713555650 x+$ $221185885116625=0$

$N=53: \quad x^{20}-1645 x^{18}-55390 x^{17}-954799 x^{16}-10534980 x^{15}-80328730 x^{14}-432066160 x^{13}-1580782544 x^{12}-$ $3165203030 x^{11}+3001160245 x^{10}+51388082540 x^{9}+222946040956 x^{8}+619309139200 x^{7}+1241295445680 x^{6}+1849999181760 x^{5}+$ $2046327491136 x^{4}+1639179074560 x^{3}+901564467200 x^{2}+305209344000 x+48015360000=0$

$N=57: \quad x^{20}-1640 x^{18}-55750 x^{17}-979889 x^{16}-11222490 x^{15}-91786180 x^{14}-563367270 x^{13}-2675585364 x^{12}-$ $10025424760 x^{11}-29971534745 x^{10}-71754108660 x^{9}-136992319909 x^{8}-205807875510 x^{7}-237244065510 x^{6}-$ $200832914170 x^{5}-114805187039 x^{4}-35432987640 x^{3}+786785275 x^{2}+4505156250 x+1071875000=0$

$N=58: \quad x^{20}-1255 x^{18}-34720 x^{17}-439879 x^{16}-2522680 x^{15}+6910365 x^{14}+273223560 x^{13}+2801719911 x^{12}+$ $18202294080 x^{11}+85711897835 x^{10}+306901177840 x^{9}+852773965811 x^{8}+1851455169480 x^{7}+3133403682655 x^{6}+$ 
$4089667985320 x^{5}+4032693050156 x^{4}+2901379923120 x^{3}+1434673598400 x^{2}+435047328000 x+60860160000=0$ $N=61: \quad x^{20}-1010 x^{18}-26850 x^{17}-366049 x^{16}-3188350 x^{15}-19039685 x^{14}-78500670 x^{13}-205873884 x^{12}-$ $184866190 x^{11}+1116613175 x^{10}+6532251540 x^{9}+19786148436 x^{8}+41336345280 x^{7}+63550610320 x^{6}+73002556480 x^{5}+$ $62158152896 x^{4}+38150517760 x^{3}+15968819200 x^{2}+4079616000 x+479641600=0$

$N=74: \quad x^{20}-460 x^{18}-6500 x^{17}-22334 x^{16}+305440 x^{15}+4600200 x^{14}+31832820 x^{13}+142409341 x^{12}+$ $449681120 x^{11}+1034766980 x^{10}+1748059760 x^{9}+2146572416 x^{8}+1862653440 x^{7}+1080161280 x^{6}+374353920 x^{5}+$ $58392576 x^{4}=0$

$N=77: \quad x^{20}-350 x^{18}-5170 x^{17}-35834 x^{16}-124900 x^{15}-7135 x^{14}+2520890 x^{13}+16883531 x^{12}+72293870 x^{11}+$ $239860145 x^{10}+653346830 x^{9}+1489750511 x^{8}+2863693610 x^{7}+4640269940 x^{6}+6245004450 x^{5}+6696282416 x^{4}+$ $5284917420 x^{3}+2671412400 x^{2}+635418000 x=0$

$N=79: \quad x^{20}-460 x^{18}-8160 x^{17}-73399 x^{16}-414860 x^{15}-1562550 x^{14}-3811990 x^{13}-4583789 x^{12}+$ $5757640 x^{11}+42935740 x^{10}+114455960 x^{9}+199943696 x^{8}+253818400 x^{7}+241426160 x^{6}+172771840 x^{5}+91820416 x^{4}+$ $35128320 x^{3}+9122560 x^{2}+1433600 x+102400=0$

$N=82: \quad x^{20}-445 x^{18}-7000 x^{17}-47234 x^{16}-107800 x^{15}+687710 x^{14}+7371400 x^{13}+34652421 x^{12}+$ $104394600 x^{11}+219198415 x^{10}+328609200 x^{9}+350769356 x^{8}+260213200 x^{7}+127264320 x^{6}+36806400 x^{5}+4755456 x^{4}=$ 0

$N=83: \quad x^{20}-395 x^{18}-6640 x^{17}-57844 x^{16}-326370 x^{15}-1286005 x^{14}-3597890 x^{13}-6788914 x^{12}-$ $6242550 x^{11}+8891645 x^{10}+50841850 x^{9}+119430631 x^{8}+190938960 x^{7}+227676805 x^{6}+207394280 x^{5}+143831976 x^{4}+$ $74109960 x^{3}+26921700 x^{2}+6188400 x+680400=0$

$N=86: \quad x^{20}-290 x^{18}-3280 x^{17}-8934 x^{16}+106400 x^{15}+1333210 x^{14}+7952070 x^{13}+31754076 x^{12}+$ $93400450 x^{11}+211188325 x^{10}+375191700 x^{9}+529255496 x^{8}+594426850 x^{7}+529604455 x^{6}+370566360 x^{5}+200075436 x^{4}+$ $80994400 x^{3}+23402400 x^{2}+4376250 x+407025=0$

$N=89: \quad x^{20}-340 x^{18}-5000 x^{17}-36099 x^{16}-156570 x^{15}-419935 x^{14}-605120 x^{13}+56886 x^{12}+2322990 x^{11}+$ $5452715 x^{10}+7039540 x^{9}+5641796 x^{8}+2748480 x^{7}+714960 x^{6}+60480 x^{5}-5184 x^{4}=0$

$N=91: \quad x^{20}-460 x^{18}-7750 x^{17}-63059 x^{16}-301150 x^{15}-862660 x^{14}-1301490 x^{13}-219269 x^{12}+2563470 x^{11}+$ $4037625 x^{10}+4150180 x^{9}+6133761 x^{8}-2799890 x^{7}-27971930 x^{6}-18397140 x^{5}+31939766 x^{4}+26507870 x^{3}-$ $17847875 x^{2}-9872500 x+4312500=0$

$N=101: \quad x^{20}-170 x^{18}-1570 x^{17}-5544 x^{16}-350 x^{15}+73120 x^{14}+318300 x^{13}+732666 x^{12}+1023690 x^{11}+$ $806400 x^{10}+151110 x^{9}-367129 x^{8}-381780 x^{7}-128680 x^{6}+28960 x^{5}+38256 x^{4}+11840 x^{3}+1280 x^{2}=0$

$N=102: \quad x^{20}-255 x^{18}-2940 x^{17}-13079 x^{16}-4550 x^{15}+218470 x^{14}+1102650 x^{13}+2708736 x^{12}+3514230 x^{11}+$ $1198225 x^{10}-3810680 x^{9}-7317539 x^{8}-5820210 x^{7}-622740 x^{6}+3517170 x^{5}+3644281 x^{4}+1537330 x^{3}+183900 x^{2}-$ $33000 x=0$

$N=111: \quad x^{20}-340 x^{18}-3980 x^{17}-13204 x^{16}+54550 x^{15}+547525 x^{14}+1114300 x^{13}-3637899 x^{12}-$ $22592660 x^{11}-33956305 x^{10}+53177040 x^{9}+326180156 x^{8}+727739320 x^{7}+843000220 x^{6}-928875360 x^{5}-7196709904 x^{4}-$ $16472977760 x^{3}-19924478800 x^{2}-12693144000 x-3346440000=0$

$N=114: \quad x^{20}-150 x^{18}-1030 x^{17}+161 x^{16}+30990 x^{15}+165340 x^{14}+414930 x^{13}+457816 x^{12}-189360 x^{11}-$ $1114005 x^{10}-953420 x^{9}+217181 x^{8}+557650 x^{7}+65290 x^{6}+180270 x^{5}+392841 x^{4}+36720 x^{3}-200475 x^{2}-60750 x=0$

$N=118: \quad x^{20}-175 x^{18}-1760 x^{17}-7769 x^{16}-13220 x^{15}+33830 x^{14}+280440 x^{13}+923736 x^{12}+2009490 x^{11}+$ $3238090 x^{10}+4032200 x^{9}+3894406 x^{8}+2830430 x^{7}+1447855 x^{6}+460020 x^{5}+67626 x^{4}=0$

$N=123: \quad x^{20}-100 x^{18}-740 x^{17}-2194 x^{16}-540 x^{15}+19090 x^{14}+77490 x^{13}+169151 x^{12}+231730 x^{11}+$ $185585 x^{10}+35340 x^{9}-103274 x^{8}-125190 x^{7}-53365 x^{6}+12910 x^{5}+26066 x^{4}+12000 x^{3}+2040 x^{2}=0$

$N=131: \quad x^{20}-80 x^{18}-600 x^{17}-2134 x^{16}-3350 x^{15}+5155 x^{14}+47700 x^{13}+158016 x^{12}+349890 x^{11}+$ $579805 x^{10}+747250 x^{9}+758066 x^{8}+603320 x^{7}+370245 x^{6}+169100 x^{5}+53776 x^{4}+10440 x^{3}+900 x^{2}=0$

$N=138: \quad x^{20}-145 x^{18}-1050 x^{17}-1479 x^{16}+10940 x^{15}+43435 x^{14}+5490 x^{13}-218949 x^{12}-253560 x^{11}+$ $476145 x^{10}+905170 x^{9}-506069 x^{8}-1536900 x^{7}+234385 x^{6}+1432590 x^{5}-9504 x^{4}-708480 x^{3}-17820 x^{2}+145800 x=0$ $N=141: \quad x^{20}-80 x^{18}-450 x^{17}-654 x^{16}+2120 x^{15}+10510 x^{14}+18420 x^{13}+13676 x^{12}-2190 x^{11}-13830 x^{10}-$ $16340 x^{9}-13919 x^{8}-5880 x^{7}+3000 x^{6}+4320 x^{5}+1296 x^{4}=0$

$N=142: \quad x^{20}-130 x^{18}-970 x^{17}-2279 x^{16}+3340 x^{15}+28755 x^{14}+53330 x^{13}+6741 x^{12}-101180 x^{11}-$ $113805 x^{10}-2050 x^{9}+68301 x^{8}+90740 x^{7}+80065 x^{6}-56410 x^{5}-105114 x^{4}+23900 x^{3}+39515 x^{2}-12300 x-450=0$ $N=143: \quad x^{20}-70 x^{18}-450 x^{17}-1154 x^{16}+160 x^{15}+10290 x^{14}+32480 x^{13}+45411 x^{12}+10450 x^{11}-43545 x^{10}+$ $39490 x^{9}+343461 x^{8}+598920 x^{7}+471275 x^{6}+129130 x^{5}-34569 x^{4}-16380 x^{3}+2700 x^{2}=0$

$N=159: \quad x^{20}-100 x^{18}-670 x^{17}-1864 x^{16}-2580 x^{15}-2755 x^{14}-5470 x^{13}-7154 x^{12}+1420 x^{11}+9130 x^{10}+$ $3350 x^{9}+6496 x^{8}+21400 x^{7}+13005 x^{6}-11010 x^{5}-15879 x^{4}-6440 x^{3}-880 x^{2}=0$

$N=174: \quad x^{20}-70 x^{18}-520 x^{17}-1954 x^{16}-4300 x^{15}-3120 x^{14}+15780 x^{13}+75201 x^{12}+184980 x^{11}+$ $312350 x^{10}+348100 x^{9}+200456 x^{8}-110760 x^{7}-401360 x^{6}-402560 x^{5}-181504 x^{4}-30720 x^{3}=0$

$N=182: \quad x^{20}-50 x^{18}-230 x^{17}-149 x^{16}+2210 x^{15}+10460 x^{14}+25750 x^{13}+41651 x^{12}+46150 x^{11}+32875 x^{10}+$ 
$8940 x^{9}-12459 x^{8}-21930 x^{7}-17580 x^{6}-6860 x^{5}+456 x^{4}+1870 x^{3}+795 x^{2}+100 x=0$

$N=222: \quad x^{20}-40 x^{18}-140 x^{17}+106 x^{16}+1690 x^{15}+4035 x^{14}+2220 x^{13}-7229 x^{12}-14380 x^{11}-3625 x^{10}+$

$15980 x^{9}+15986 x^{8}-4560 x^{7}-12420 x^{6}-2160 x^{5}+4536 x^{4}=0$

$N=231: \quad x^{20}-20 x^{18}-130 x^{17}-314 x^{16}-280 x^{15}+980 x^{14}+3380 x^{13}+4961 x^{12}+380 x^{11}-7900 x^{10}-$

$13570 x^{9}-2884 x^{8}+9380 x^{7}+14240 x^{6}-4080 x^{5}-3664 x^{4}-480 x^{3}=0$

$N=238: \quad x^{20}-25 x^{18}-230 x^{17}-1209 x^{16}-4720 x^{15}-14295 x^{14}-35010 x^{13}-68204 x^{12}-98940 x^{11}-85840 x^{10}+$

$45260 x^{9}+367756 x^{8}+862040 x^{7}+1394440 x^{6}+1738480 x^{5}+1610256 x^{4}+1002720 x^{3}+364320 x^{2}+57600 x=0$

$m=7$ :

$N=37: \quad x^{28}-41860 x^{26}-6127954 x^{25}-427432495 x^{24}-18550325528 x^{23}-560710780081 x^{22}-12552197804790 x^{21}-$ $215828540749521 x^{20}-2910419425162774 x^{19}-31030929091681001 x^{18}-259779656065288954 x^{17}-1647986021397462160 x^{16}-$ $6971675325797235466 x^{15}-6545057267939397935 x^{14}+193181926295015715470 x^{13}+2082026545962578174421 x^{12}+$ $13515120338930746212222 x^{11}+65612617796717805260317 x^{10}+252501457334175006170584 x^{9}+786076612450217262431607 x^{8}+$ $1989090793456322295375342 x^{7}+4072080386883072232720548 x^{6}+6658957039653444844422972 x^{5}+8506684347590576662124499$ $8188017016238518491941580 x^{3}+5588434756492068796677180 x^{2}+2411749190962684641460896 x+494943386251579331532480=$ 0

$N=43: \quad x^{28}-19180 x^{26}-2061920 x^{25}-111083994 x^{24}-3841249538 x^{23}-94452217087 x^{22}-1747024126722 x^{21}-$ $25156014494653 x^{20}-288049203825474 x^{19}-2653528947881238 x^{18}-19714153185812890 x^{17}-116959315017515678 x^{16}-$ $535361926432968138 x^{15}-1691826986690641703 x^{14}-1792467522812480990 x^{13}+18906857045085538179 x^{12}+160374234341419592$ $769529277816008248325 x^{10}+2719508638124373935112 x^{9}+7545180553898574020140 x^{8}+16764750980289631701928 x^{7}+$ $29890779523422316342662 x^{6}+42364025726777007413076 x^{5}+46763926726175916349149 x^{4}+38818709594021419600230 x^{3}+$ $22818673617622961341725 x^{2}+8473367948492204430750 x+1495113591495589065000=0$

$N=53: \quad x^{28}-6328 x^{26}-394590 x^{25}-12348896 x^{24}-246622180 x^{23}-3448782656 x^{22}-35180530070 x^{21}-$ $262911261949 x^{20}-1362058319272 x^{19}-3554774952463 x^{18}+14011361556472 x^{17}+242015838630992 x^{16}+1751404427123120 x^{15}+$ $8970827026795039 x^{14}+36032358501525218 x^{13}+117915766781709435 x^{12}+319884912054504272 x^{11}+724913490963978116 x^{10}+$ $1374882502722820742 x^{9}+2176984487728213373 x^{8}+2859040963286671604 x^{7}+3080188917248563435 x^{6}+2677053726503258060 x^{5}$ $1830728471348857627 x^{4}+948232678909261088 x^{3}+349517436207775017 x^{2}+81643949573627392 x+9077517857311113=$ 0

$N=57: \quad x^{28}-5488 x^{26}-335258 x^{25}-10479399 x^{24}-210856366 x^{23}-2981934792 x^{22}-30953684286 x^{21}-$ $240764236251 x^{20}-1407157536414 x^{19}-6052866808275 x^{18}-17770008886854 x^{17}-25225634988807 x^{16}+54328574869254 x^{15}+$ $443356258978941 x^{14}+1348434826781826 x^{13}+2225404583429202 x^{12}+882338209238598 x^{11}-5587411122930529 x^{10}$ $16833627885709656 x^{9}-26401873186479350 x^{8}-26522973791185312 x^{7}-17141814692501457 x^{6}-6351352292111372 x^{5}-$ $705761579123796 x^{4}+345899475361440 x^{3}+103460783025600 x^{2}=0$

$N=58: \quad x^{28}-4424 x^{26}-225288 x^{25}-5273821 x^{24}-59012534 x^{23}+114636805 x^{22}+16465631014 x^{21}+$ $329973454398 x^{20}+4158848097950 x^{19}+38826402600587 x^{18}+285292125532554 x^{17}+1701937136656798 x^{16}+8395979271226372 x^{15}$ $34649314339126578 x^{14}+120490919030065052 x^{13}+354509220902823917 x^{12}+883852634187690972 x^{11}+1865815930065573678 x^{10}+$ $3324630912138407788 x^{9}+4972211098981956599 x^{8}+6187612078855733330 x^{7}+6327356791813230201 x^{6}+5222659938725033630 x^{5}$ $3390968286286695108 x^{4}+1665953654353415910 x^{3}+581586030482500575 x^{2}+128418213273635250 x+13466030721255000=$ 0

$N=61: \quad x^{28}-3444 x^{26}-165200 x^{25}-4050452 x^{24}-63955640 x^{23}-708393431 x^{22}-5692535758 x^{21}-$ $32934019769 x^{20}-124939989692 x^{19}-151010971202 x^{18}+1854338995810 x^{17}+17418154123054 x^{16}+92191149483104 x^{15}+$ $360003340030439 x^{14}+1114983395174488 x^{13}+2821627870272596 x^{12}+5916666257686310 x^{11}+10342847028907039 x^{10}+$ $15088156094743578 x^{9}+18313680444201835 x^{8}+18369500162149444 x^{7}+15057300508187448 x^{6}+9918038548201770 x^{5}+$ $5120388873755100 x^{4}+1994600057042250 x^{3}+550937284419375 x^{2}+96118066800000 x+7958527171875=0$

$N=65: \quad x^{28}-2639 x^{26}-114184 x^{25}-2568426 x^{24}-37676898 x^{23}-390604491 x^{22}-2943548818 x^{21}-$ $15908911473 x^{20}-55822203794 x^{19}-61454760521 x^{18}+666600371486 x^{17}+5354836889779 x^{16}+22999234873874 x^{15}+$ $67410933208948 x^{14}+137827466221242 x^{13}+177275513152633 x^{12}+62010380553562 x^{11}-278992175129063 x^{10}-$ $653051345528806 x^{9}-628412389420130 x^{8}-20819711392536 x^{7}+686838187643335 x^{6}+793746804542160 x^{5}+278133722654103 x^{4}-$ $215640988837344 x^{3}-285823371944817 x^{2}-127679637259944 x-21707500207239=0$

$N=74: \quad x^{28}-1414 x^{26}-37170 x^{25}-340445 x^{24}+1778868 x^{23}+88347339 x^{22}+1272396174 x^{21}+11735045357 x^{20}+$ $79745012634 x^{19}+422842081403 x^{18}+1803883729410 x^{17}+6306861239230 x^{16}+18283763023806 x^{15}+44272580103843 x^{14}+$ $89906688853674 x^{13}+153336645311633 x^{12}+219389803139430 x^{11}+262351027460343 x^{10}+260440719171780 x^{9}+$ $212378186704915 x^{8}+140046720669618 x^{7}+72969349643218 x^{6}+29007902441700 x^{5}+8320646704861 x^{4}+1560634852812 x^{3}+$ $155072494276 x^{2}+3928804320 x+29042496=0$

$N=79: \quad x^{28}-1288 x^{26}-39144 x^{25}-616609 x^{24}-6290452 x^{23}-44822467 x^{22}-227119144 x^{21}-781694172 x^{20}-$ $1374567572 x^{19}+2704465407 x^{18}+31439215640 x^{17}+136902422096 x^{16}+406344933344 x^{15}+915159979944 x^{14}+$ 
$1624352525824 x^{13}+2308182688224 x^{12}+2640194070080 x^{11}+2429014833136 x^{10}+1787209574528 x^{9}+1040612761216 x^{8}+$ $471699053056 x^{7}+162430809856 x^{6}+40929079296 x^{5}+7098015744 x^{4}+755564544 x^{3}+37158912 x^{2}=0$

$N=82: \quad x^{28}-1183 x^{26}-32592 x^{25}-424207 x^{24}-2745988 x^{23}+1234663 x^{22}+222719952 x^{21}+2653629265 x^{20}+$ $19687018862 x^{19}+108296030430 x^{18}+470062667102 x^{17}+1660902741707 x^{16}+4865566841254 x^{15}+11956773850567 x^{14}+$ $24844046134180 x^{13}+43894774110257 x^{12}+66238125017330 x^{11}+85707086570339 x^{10}+95449214729918 x^{9}+91773898057954 x^{8}+$ $76227550249438 x^{7}+54405862738416 x^{6}+32857071964528 x^{5}+16304961741055 x^{4}+6342675513328 x^{3}+1797850628000 x^{2}+$ $327379922688 x+28539092736=0$

$N=83: \quad x^{28}-1064 x^{26}-30492 x^{25}-465962 x^{24}-4768036 x^{23}-35551534 x^{22}-200431028 x^{21}-860215755 x^{20}-$ $2716323456 x^{19}-5355716660 x^{18}+646440340 x^{17}+57370263901 x^{16}+284826995740 x^{15}+926838113110 x^{14}+2326545031748 x^{13}+$ $4741828887698 x^{12}+8015067903080 x^{11}+11334197202408 x^{10}+13434048072648 x^{9}+13306274175173 x^{8}+10933944801696 x^{7}+$ $7365714246156 x^{6}+3997051548336 x^{5}+1703026570176 x^{4}+548217573120 x^{3}+125316289728 x^{2}+18139790592 x+$ $1252806912=0$

$N=86: \quad x^{28}-812 x^{26}-16408 x^{25}-112742 x^{24}+620522 x^{23}+21166953 x^{22}+240431534 x^{21}+1775825751 x^{20}+$ $9720640258 x^{19}+41646979542 x^{18}+143858429218 x^{17}+408051973202 x^{16}+962077874926 x^{15}+1901189606981 x^{14}+$ $3166471900662 x^{13}+4459863541091 x^{12}+5319372845976 x^{11}+5369197797757 x^{10}+4572878095296 x^{9}+3267333091456 x^{8}+$ $1939933638076 x^{7}+943066577530 x^{6}+366913548400 x^{5}+110213153625 x^{4}+24060951250 x^{3}+3403640625 x^{2}+234281250 x=$ 0

$N=89: \quad x^{28}-854 x^{26}-21616 x^{25}-288638 x^{24}-2549484 x^{23}-16167673 x^{22}-75974766 x^{21}-262787763 x^{20}-$ $617016134 x^{19}-579623996 x^{18}+2645887636 x^{17}+16895740189 x^{16}+56956652242 x^{15}+140834365868 x^{14}+276837285648 x^{13}+$ $446777420950 x^{12}+601234618404 x^{11}+680012344399 x^{10}+648910849944 x^{9}+523195135281 x^{8}+356243635314 x^{7}+$ $204251953129 x^{6}+97864436712 x^{5}+38530733172 x^{4}+12052155402 x^{3}+2811249000 x^{2}+432885600 x+32772033=0$

$N=101: \quad x^{28}-420 x^{26}-7028 x^{25}-57596 x^{24}-263606 x^{23}-426892 x^{22}+3175074 x^{21}+30681791 x^{20}+$ $150976378 x^{19}+531234886 x^{18}+1464494934 x^{17}+3299643465 x^{16}+6228261004 x^{15}+10010416710 x^{14}+13854204588 x^{13}+$ $16635091424 x^{12}+17406360930 x^{11}+15894778910 x^{10}+12644670346 x^{9}+8716631175 x^{8}+5157176486 x^{7}+2580057046 x^{6}+$ $1067733926 x^{5}+353936332 x^{4}+89518408 x^{3}+15982960 x^{2}+1756160 x+87808=0$

$N=102: \quad x^{28}-602 x^{26}-11284 x^{25}-96243 x^{24}-355320 x^{23}+1171131 x^{22}+24940944 x^{21}+180249412 x^{20}+$ $834021734 x^{19}+2709394954 x^{18}+6117136256 x^{17}+8294819441 x^{16}+594295688 x^{15}-26735947476 x^{14}-66130948338 x^{13}-$ $78086128526 x^{12}-19736510892 x^{11}+87045453631 x^{10}+144432250578 x^{9}+89344360217 x^{8}-14361695320 x^{7}-64152663505 x^{6}-$ $48307735518 x^{5}-18341608081 x^{4}-3541687506 x^{3}-180319125 x^{2}-2376990 x-9261=0$

$N=111: \quad x^{28}-700 x^{26}-12754 x^{25}-87829 x^{24}-60662 x^{23}+2757926 x^{22}+14913444 x^{21}-1286649 x^{20}-$ $279761692 x^{19}-853613432 x^{18}+1243785494 x^{17}+12665022173 x^{16}+18317460098 x^{15}-62324105522 x^{14}-258067720456 x^{13}-$ $138003879552 x^{12}+1062097301328 x^{11}+2529262169248 x^{10}+491311100224 x^{9}-7011140664528 x^{8}-12703190203872 x^{7}-$ $5453279953632 x^{6}+12531109909248 x^{5}+24565320479232 x^{4}+21100913164800 x^{3}+10161562586112 x^{2}+2650098124800 x+$ $290358577152=0$

$N=114: \quad x^{28}-322 x^{26}-4298 x^{25}-17269 x^{24}+135394 x^{23}+2253436 x^{22}+14649138 x^{21}+55166125 x^{20}+$ $121280474 x^{19}+112406007 x^{18}-139564334 x^{17}-523138099 x^{16}-448799330 x^{15}+314970803 x^{14}+786456650 x^{13}+$ $172458930 x^{12}-427771666 x^{11}-54417297 x^{10}+165938304 x^{9}-160701660 x^{8}-41277096 x^{7}+78306669 x^{6}-53068932 x^{5}+$ $34046244 x^{4}-10287648 x^{3}+979776 x^{2}=0$

$N=118: \quad x^{28}-378 x^{26}-6104 x^{25}-48496 x^{24}-210588 x^{23}-207820 x^{22}+4110708 x^{21}+36348858 x^{20}+$ $186465524 x^{19}+708513680 x^{18}+2145862516 x^{17}+5353992552 x^{16}+11195006284 x^{15}+19792802764 x^{14}+29693069980 x^{13}+$ $37768433661 x^{12}+40531746108 x^{11}+36349414090 x^{10}+26821158420 x^{9}+15894211536 x^{8}+7282789920 x^{7}+2422641312 x^{6}+$ $520043328 x^{5}+53934336 x^{4}=0$

$N=123: \quad x^{28}-210 x^{26}-2968 x^{25}-20426 x^{24}-71120 x^{23}-21893 x^{22}+1059142 x^{21}+5927992 x^{20}+18507762 x^{19}+$ $38282664 x^{18}+52139906 x^{17}+36806433 x^{16}-18719750 x^{15}-89521537 x^{14}-125131160 x^{13}-94289874 x^{12}-16324994 x^{11}+$ $56545163 x^{10}+83792842 x^{9}+63133567 x^{8}+22566082 x^{7}-7232246 x^{6}-14997780 x^{5}-9335276 x^{4}-2888032 x^{3}-$ $285824 x^{2}+65856 x+15680=0$

$N=130: \quad x^{28}-231 x^{26}-1316 x^{25}+12642 x^{24}+158522 x^{23}+426569 x^{22}-1816682 x^{21}-13095033 x^{20}-$ $10871322 x^{19}+116360839 x^{18}+312370226 x^{17}-401334753 x^{16}-2686622274 x^{15}-1207521784 x^{14}+12348475986 x^{13}+$ $20580977697 x^{12}-22413901370 x^{11}-90714598475 x^{10}-36683952634 x^{9}+161233022258 x^{8}+209337731232 x^{7}-58976761473 x^{6}-$ $272958077056 x^{5}-112740012377 x^{4}+118752090044 x^{3}+98578489051 x^{2}-5963470716 x-16488077571=0$

$N=131: \quad x^{28}-182 x^{26}-2170 x^{25}-13230 x^{24}-47110 x^{23}-68485 x^{22}+286412 x^{21}+2484160 x^{20}+10427620 x^{19}+$ $31561068 x^{18}+75792556 x^{17}+150311055 x^{16}+251229944 x^{15}+357689850 x^{14}+435859494 x^{13}+454655474 x^{12}+$ $404408298 x^{11}+304184933 x^{10}+190811740 x^{9}+97671980 x^{8}+39416384 x^{7}+11835824 x^{6}+2367680 x^{5}+238400 x^{4}=0$ $N=138: \quad x^{28}-266 x^{26}-3248 x^{25}-18053 x^{24}-43428 x^{23}+103561 x^{22}+1401232 x^{21}+6433077 x^{20}+$ $17783318 x^{19}+30363221 x^{18}+23016728 x^{17}-29476959 x^{16}-114165590 x^{15}-153390853 x^{14}-63201908 x^{13}+127730414 x^{12}+$ $256189962 x^{11}+186183273 x^{10}-22621424 x^{9}-172850832 x^{8}-154368214 x^{7}-41383160 x^{6}+37319212 x^{5}+42980176 x^{4}+$ 
$18693360 x^{3}+3326400 x^{2}=0$

$N=141: \quad x^{28}-140 x^{26}-1694 x^{25}-9814 x^{24}-27888 x^{23}+612 x^{22}+351106 x^{21}+1665209 x^{20}+4699814 x^{19}+$ $9418094 x^{18}+14046480 x^{17}+15474605 x^{16}+11475128 x^{15}+2990904 x^{14}-6026874 x^{13}-11450131 x^{12}-11812934 x^{11}-$ $8632530 x^{10}-4525388 x^{9}-1329559 x^{8}+407652 x^{7}+954884 x^{6}+831558 x^{5}+494964 x^{4}+214578 x^{3}+66150 x^{2}+13230 x+$ $1323=0$

$N=142: \quad x^{28}-259 x^{26}-2940 x^{25}-12691 x^{24}-1204 x^{23}+234979 x^{22}+1131396 x^{21}+2400188 x^{20}+$ $800380 x^{19}-8815926 x^{18}-22224552 x^{17}-14213942 x^{16}+37609880 x^{15}+90119974 x^{14}+39498088 x^{13}-116074007 x^{12}-$ $175008680 x^{11}+151329 x^{10}+189771988 x^{9}+121252537 x^{8}-72887780 x^{7}-106652217 x^{6}-9339372 x^{5}+34807914 x^{4}+$ $12367404 x^{3}-3197880 x^{2}-1714608 x=0$

$N=143: \quad x^{28}-154 x^{26}-1512 x^{25}-6524 x^{24}-8862 x^{23}+50354 x^{22}+330932 x^{21}+939463 x^{20}+1401820 x^{19}+$ $518560 x^{18}-1751162 x^{17}-1168973 x^{16}+9448698 x^{15}+31198986 x^{14}+52008376 x^{13}+55104896 x^{12}+37200240 x^{11}+$ $13092614 x^{10}-674058 x^{9}-1710863 x^{8}+1443288 x^{7}+2337720 x^{6}+1192576 x^{5}+234256 x^{4}=0$

$N=145: \quad x^{28}-245 x^{26}-2688 x^{25}-11718 x^{24}-13538 x^{23}+68673 x^{22}+245476 x^{21}+35462 x^{20}-984354 x^{19}-$ $956473 x^{18}+2054220 x^{17}+2574466 x^{16}-3189480 x^{15}-1797880 x^{14}+6142850 x^{13}-4560227 x^{12}-10772986 x^{11}+$ $14590936 x^{10}+10796940 x^{9}-19913320 x^{8}-3113390 x^{7}+15232525 x^{6}-1167950 x^{5}-4164600 x^{4}+1102500 x^{3}=0$

$N=155: \quad x^{28}-112 x^{26}-1120 x^{25}-5796 x^{24}-16730 x^{23}-10812 x^{22}+144298 x^{21}+800576 x^{20}+2384340 x^{19}+$ $4530365 x^{18}+4807586 x^{17}-602400 x^{16}-11737054 x^{15}-19569963 x^{14}-12106696 x^{13}+8987874 x^{12}+24316166 x^{11}+$ $18046388 x^{10}-1587754 x^{9}-13182554 x^{8}-9153480 x^{7}-283815 x^{6}+2973082 x^{5}+1385175 x^{4}-81242 x^{3}-128051 x^{2}+$ $58604 x+33124=0$

$N=159: \quad x^{28}-175 x^{26}-1974 x^{25}-11291 x^{24}-41146 x^{23}-102281 x^{22}-165746 x^{21}-123403 x^{20}+126140 x^{19}+$ $454559 x^{18}+561946 x^{17}+423863 x^{16}+284900 x^{15}+173299 x^{14}+68810 x^{13}+308007 x^{12}+1208858 x^{11}+2651561 x^{10}+$ $4207490 x^{9}+5379815 x^{8}+5816384 x^{7}+5479957 x^{6}+4562726 x^{5}+3291016 x^{4}+1942220 x^{3}+862848 x^{2}+254800 x+37632=$ 0

$N=174: \quad x^{28}-140 x^{26}-1386 x^{25}-7168 x^{24}-20468 x^{23}-6458 x^{22}+215824 x^{21}+1069320 x^{20}+3057656 x^{19}+$ $6319712 x^{18}+10528056 x^{17}+15695161 x^{16}+22970752 x^{15}+33595716 x^{14}+46169690 x^{13}+55615952 x^{12}+56308476 x^{11}+$ $46896552 x^{10}+31680880 x^{9}+17090864 x^{8}+7197344 x^{7}+2274048 x^{6}+498176 x^{5}+61440 x^{4}=0$

$N=190: \quad x^{28}-133 x^{26}-1120 x^{25}-3577 x^{24}+896 x^{23}+44341 x^{22}+134512 x^{21}+75642 x^{20}-511504 x^{19}-$ $1382514 x^{18}-870016 x^{17}+2531262 x^{16}+6016640 x^{15}+2089626 x^{14}-9295776 x^{13}-11152491 x^{12}+4589536 x^{11}+$ $13941399 x^{10}+2358048 x^{9}-8427125 x^{8}-4285568 x^{7}+3055073 x^{6}+2445744 x^{5}-1111648 x^{4}-534352 x^{3}+340144 x^{2}-$ $47040 x=0$

$N=195: \quad x^{28}-98 x^{26}-994 x^{25}-5481 x^{24}-19866 x^{23}-47436 x^{22}-64288 x^{21}+10227 x^{20}+261898 x^{19}+$ $607663 x^{18}+711788 x^{17}+264739 x^{16}-495586 x^{15}-1353254 x^{14}-1831074 x^{13}-107114 x^{12}+5094418 x^{11}+7285393 x^{10}-$ $5938408 x^{9}-12990404 x^{8}+627312 x^{7}+6245764 x^{6}+1654800 x^{5}+90000 x^{4}=0$

$N=222: \quad x^{28}-70 x^{26}-378 x^{25}+133 x^{24}+8442 x^{23}+29046 x^{22}+2184 x^{21}-193837 x^{20}-352044 x^{19}+283262 x^{18}+$ $1532454 x^{17}+961519 x^{16}-2471742 x^{15}-4165854 x^{14}+380940 x^{13}+6319880 x^{12}+4325328 x^{11}-4348656 x^{10}-7107072 x^{9}+$ $369712 x^{8}+5941152 x^{7}+2034592 x^{6}-2796864 x^{5}-1931264 x^{4}+387072 x^{3}+641536 x^{2}+150528 x=0$

$m=11:$

$N=210: \quad x^{44}-363 x^{42}-3696 x^{41}+3630 x^{40}+234520 x^{39}+901681 x^{38}-4647082 x^{37}-40273959 x^{36}-$ $7112996 x^{35}+757600631 x^{34}+1771143990 x^{33}-7115440101 x^{32}-33445549456 x^{31}+22326485071 x^{30}+331532157022 x^{29}+$ $232898882458 x^{28}-2017194264756 x^{27}-3416497782016 x^{26}+7538291692954 x^{25}+22031278885611 x^{24}-13683397029456 x^{23}-$ $87242784188780 x^{22}-16366424999188 x^{21}+216854815308296 x^{20}+168631250764064 x^{19}-288523782910176 x^{18}-$ $420494177868160 x^{17}+16191775557584 x^{16}+340048209314880 x^{15}+526928872258752 x^{14}+504609469876160 x^{13}-$ $564193502139520 x^{12}-1401796570028800 x^{11}-96484826380800 x^{10}+1272997503360000 x^{9}+496652504832000 x^{8}-$ $519417930240000 x^{7}-294585984000000 x^{6}+79651123200000 x^{5}+55539302400000 x^{4}=0$

Appendix C. Numerical values of the generators $x_{N}$ And $y_{N}$ AT the CM Point $z=i \sqrt{m / N}$

For all positive square-free levels $N$ such that $\Gamma_{0}(N)^{+}$has genus one, and some small primes $m$, we list here numerical values of the generators $x_{N}$ and $y_{N}$ at the CM point $z=i \sqrt{m / N}$.

$x_{37}(i \sqrt{2 / 37})=30.84491025792583$

$x_{43}(i \sqrt{2 / 43})=26.03830445619595$

$x_{53}(i \sqrt{2 / 53})=17.14080423535380$

$x_{57}(i \sqrt{2 / 57})=19.82475165290612$ $y_{37}(i \sqrt{2 / 37})=101.4880189467247$

$y_{43}(i \sqrt{2 / 43})=72.73532975449962$

$y_{53}(i \sqrt{2 / 53})=55.03697013319314$

$y_{57}(i \sqrt{2 / 57})=41.64950330581225$ 
$x_{61}(i \sqrt{2 / 61})=14.87419633124004$

$x_{65}(i \sqrt{2 / 65})=14.00000000000000$

$x_{77}(i \sqrt{2 / 77})=8.690415759823430$

$x_{79}(i \sqrt{2 / 79})=11.79530756303855$

$x_{83}(i \sqrt{2 / 83})=11.30927278350279$

$x_{89}(i \sqrt{2 / 89})=10.78162836940127$

$x_{91}(i \sqrt{2 / 91})=13.66717925910431$

$x_{101}(i \sqrt{2 / 101})=6.930382822314249$

$x_{111}(i \sqrt{2 / 111})=12.28908857436736$

$x_{123}(i \sqrt{2 / 123})=5.918134420760141$

$x_{131}(i \sqrt{2 / 131})=5.682743580251222$

$x_{141}(i \sqrt{2 / 141})=5.449489742783178$

$x_{143}(i \sqrt{2 / 143})=5.409410846671381$

$x_{145}(i \sqrt{2 / 145})=7.730644941388561$

$x_{155}(i \sqrt{2 / 155})=5.162277660168379$

$x_{159}(i \sqrt{2 / 159})=7.229590467696371$

$x_{195}(i \sqrt{2 / 195})=6.162277660168379$

$x_{231}(i \sqrt{2 / 231})=4.000000000000000$

$x_{37}(i \sqrt{3 / 37})=49.97509690401588$

$x_{43}(i \sqrt{3 / 43})=40.15353988809655$

$x_{53}(i \sqrt{3 / 53})=25.76369563671823$

$x_{58}(i \sqrt{3 / 58})=22.88428673789523$

$x_{61}(i \sqrt{3 / 61})=21.54385099464021$

$x_{65}(i \sqrt{3 / 65})=19.97430038622715$

$x_{74}(i \sqrt{3 / 74})=13.28908857436736$

$x_{77}(i \sqrt{3 / 77})=12.63197666808049$

$x_{79}(i \sqrt{3 / 79})=16.08561222698957$

$x_{82}(i \sqrt{3 / 82})=15.51215751109299$

$x_{83}(i \sqrt{3 / 83})=15.27809723246796$

$x_{86}(i \sqrt{3 / 86})=11.17890834580027$

$x_{89}(i \sqrt{3 / 89})=14.32051320768432$

$x_{91}(i \sqrt{3 / 91})=17.53939201416946$

$x_{101}(i \sqrt{3 / 101})=9.432533732982306$

$x_{118}(i \sqrt{3 / 118})=10.96974774981665$

$x_{130}(i \sqrt{3 / 130})=4.162277660168379$

$x_{131}(i \sqrt{3 / 131})=7.369702609534323$

$x_{142}(i \sqrt{3 / 142})=9.587526096679698$

$x_{143}(i \sqrt{3 / 143})=6.881516951801326$

$x_{145}(i \sqrt{3 / 145})=9.459177702481585$

$x_{155}(i \sqrt{3 / 155})=6.471090946351502$

$x_{182}(i \sqrt{3 / 182})=5.747522912898956$

$x_{190}(i \sqrt{3 / 190})=7.898979485566356$

$x_{238}(i \sqrt{3 / 238})=6.382061962799605$

$x_{37}(i \sqrt{5 / 37})=122.6912619702792$

$x_{43}(i \sqrt{5 / 43})=90.69229395717788$

$x_{53}(i \sqrt{5 / 53})=55.10934173331341$

$x_{57}(i \sqrt{5 / 57})=55.21602173212197$

$x_{58}(i \sqrt{5 / 58})=47.04389740582812$

$x_{61}(i \sqrt{5 / 61})=43.26182187864434$

$x_{74}(i \sqrt{5 / 74})=26.60170461646943$

$x_{77}(i \sqrt{5 / 77})=24.94987437106620$ $y_{61}(i \sqrt{2 / 61})=41.28159030939000$

$y_{65}(i \sqrt{2 / 65})=38.00000000000000$

$y_{77}(i \sqrt{2 / 77})=33.76166303929372$

$y_{79}(i \sqrt{2 / 79})=26.06217792867651$

$y_{83}(i \sqrt{2 / 83})=24.56839217909091$

$y_{89}(i \sqrt{2 / 89})=22.29210017238130$

$y_{91}(i \sqrt{2 / 91})=18.66717925910431$

$y_{101}(i \sqrt{2 / 101})=22.01510303191421$

$y_{111}(i \sqrt{2 / 111})=14.28908857436736$

$y_{123}(i \sqrt{2 / 123})=15.51215751109299$

$y_{131}(i \sqrt{2 / 131})=14.64678729944324$

$y_{141}(i \sqrt{2 / 141})=13.34846922834953$

$y_{143}(i \sqrt{2 / 143})=13.13086285404300$

$y_{145}(i \sqrt{2 / 145})=10.66802552563710$

$y_{155}(i \sqrt{2 / 155})=12.32455532033676$

$y_{159}(i \sqrt{2 / 159})=9.759346965727467$

$y_{195}(i \sqrt{2 / 195})=6.162277660168379$

$y_{231}(i \sqrt{2 / 231})=7.000000000000000$

$y_{37}(i \sqrt{3 / 37})=237.1214938645054$

$y_{43}(i \sqrt{3 / 43})=159.7356226576774$

$y_{53}(i \sqrt{3 / 53})=105.6428939343164$

$y_{58}(i \sqrt{3 / 58})=88.84621476870907$

$y_{61}(i \sqrt{3 / 61})=76.32780733527740$

$y_{65}(i \sqrt{3 / 65})=68.05942427647228$

$y_{74}(i \sqrt{3 / 74})=58.15635429746942$

$y_{77}(i \sqrt{3 / 77})=54.73293707033676$

$y_{79}(i \sqrt{3 / 79})=44.85870034177378$

$y_{82}(i \sqrt{3 / 82})=45.86058386370626$

$y_{83}(i \sqrt{3 / 83})=41.41439970068931$

$y_{86}(i \sqrt{3 / 86})=42.71563338320109$

$y_{89}(i \sqrt{3 / 89})=36.82484416434651$

$y_{91}(i \sqrt{3 / 91})=32.07878402833891$

$y_{101}(i \sqrt{3 / 101})=33.61564935916818$

$y_{118}(i \sqrt{3 / 118})=24.71490174830570$

$y_{130}(i \sqrt{3 / 130})=24.64911064067352$

$y_{131}(i \sqrt{3 / 131})=21.41029317350333$

$y_{142}(i \sqrt{3 / 142})=19.38644241784269$

$y_{143}(i \sqrt{3 / 143})=18.94105385309107$

$y_{145}(i \sqrt{3 / 145})=16.03283656656001$

$y_{155}(i \sqrt{3 / 155})=17.34083560149767$

$y_{182}(i \sqrt{3 / 182})=12.49504582579791$

$y_{190}(i \sqrt{3 / 190})=14.34846922834953$

$y_{238}(i \sqrt{3 / 238})=9.322196990471444$

$y_{37}(i \sqrt{5 / 37})=1054.300442923472$

$y_{43}(i \sqrt{5 / 43})=637.7263594854394$

$y_{53}(i \sqrt{5 / 53})=349.4659015505589$

$y_{57}(i \sqrt{5 / 57})=273.5381815832466$

$y_{58}(i \sqrt{5 / 58})=274.2206210765973$

$y_{61}(i \sqrt{5 / 61})=234.3079793224234$

$y_{74}(i \sqrt{5 / 74})=151.3708195469660$

$y_{77}(i \sqrt{5 / 77})=138.5989949685296$ 
$x_{79}(i \sqrt{5 / 79})=29.09319310956455$

$x_{82}(i \sqrt{5 / 82})=27.63272526429506$

$x_{83}(i \sqrt{5 / 83})=27.14794662849679$

$x_{86}(i \sqrt{5 / 86})=21.0986938948931$

$x_{89}(i \sqrt{5 / 89})=24.7484285645991$

$x_{91}(i \sqrt{5 / 91})=28.65898539796081$

$x_{101}(i \sqrt{5 / 101})=16.793639129466$

$x_{102}(i \sqrt{5 / 102})=20.661903789691$

$x_{111}(i \sqrt{5 / 111})=23.04422809910841$

$x_{114}(i \sqrt{5 / 114})=14.34846922834953$

$x_{118}(i \sqrt{5 / 118})=17.42227181855$

$x_{123}(i \sqrt{5 / 123})=13.00731516491$

$x_{131}(i \sqrt{5 / 131})=12.07281873223$

$x_{138}(i \sqrt{5 / 138})=14.78232998313$

$x_{141}(i \sqrt{5 / 141})=11.11071664809$

$x_{142}(i \sqrt{5 / 142})=14.3744740037$

$x_{143}(i \sqrt{5 / 143})=10.94063877376317$

$x_{159}(i \sqrt{5 / 159})=12.8387003020$

$x_{174}(i \sqrt{5 / 174})=11.520797289$

$x_{182}(i \sqrt{5 / 182})=8.531128874149275$

$x_{222}(i \sqrt{5 / 222})=7.16227766017$

$x_{231}(i \sqrt{5 / 231})=6.790140614103718$

$x_{238}(i \sqrt{5 / 238})=8.780587274616714$

$x_{37}(i \sqrt{7 / 37})=267.9037376137941$

$x_{43}(i \sqrt{7 / 43})=185.0885481120074$

$x_{53}(i \sqrt{7 / 53})=106.54835173477$

$x_{57}(i \sqrt{7 / 57})=100.5053789785310$

$x_{58}(i \sqrt{7 / 58})=88.008293578404$

$x_{61}(i \sqrt{7 / 61})=79.449556390373$

$x_{65}(i \sqrt{7 / 65})=70.12155720683171$

$x_{74}(i \sqrt{7 / 74})=47.9418771504722$

$x_{79}(i \sqrt{7 / 79})=49.0370849107$

$x_{82}(i \sqrt{7 / 82})=46.0214022293$

$x_{83}(i \sqrt{7 / 83})=45.0743261826$

$x_{86}(i \sqrt{7 / 86})=36.3230084169$

$x_{89}(i \sqrt{7 / 89})=40.2047420870$

$x_{101}(i \sqrt{7 / 101})=27.620731451$

$x_{102}(i \sqrt{7 / 102})=32.408517907$

$x_{111}(i \sqrt{7 / 111})=33.9310118539$

$x_{114}(i \sqrt{7 / 114})=22.82475165290612$

$x_{118}(i \sqrt{7 / 118})=26.30092182$

$x_{123}(i \sqrt{7 / 123})=20.33541848$

$x_{130}(i \sqrt{7 / 130})=14.06225774829855$

$x_{131}(i \sqrt{7 / 131})=18.572075101$

$x_{138}(i \sqrt{7 / 138})=21.451547375$

$x_{141}(i \sqrt{7 / 141})=16.77512059$

$x_{142}(i \sqrt{7 / 142})=20.71346832$

$x_{143}(i \sqrt{7 / 143})=16.459591825520$

$x_{145}(i \sqrt{7 / 145})=20.19808581682744$

$x_{155}(i \sqrt{7 / 155})=14.80473022607986$

$x_{159}(i \sqrt{7 / 159})=18.0767164$

$$
\begin{aligned}
& y_{79}(i \sqrt{5 / 79})=120.7036521472076 \\
& y_{82}(i \sqrt{5 / 82})=115.9019990669251 \\
& y_{83}(i \sqrt{5 / 83})=108.0349714301628 \\
& y_{86}(i \sqrt{5 / 86})=104.709931929995 \\
& y_{89}(i \sqrt{5 / 89})=92.6190351699111 \\
& y_{91}(i \sqrt{5 / 91})=83.74604008623770 \\
& y_{101}(i \sqrt{5 / 101})=75.518036718828 \\
& y_{102}(i \sqrt{5 / 102})=69.985711369072 \\
& y_{111}(i \sqrt{5 / 111})=55.18317703383875 \\
& y_{114}(i \sqrt{5 / 114})=56.39387691339814 \\
& y_{118}(i \sqrt{5 / 118})=53.19445933649 \\
& y_{123}(i \sqrt{5 / 123})=49.14934354838 \\
& y_{131}(i \sqrt{5 / 131})=44.06918380480 \\
& y_{138}(i \sqrt{5 / 138})=40.34698994938 \\
& y_{141}(i \sqrt{5 / 141})=38.72116444264 \\
& y_{142}(i \sqrt{5 / 142})=38.4940756169 \\
& y_{143}(i \sqrt{5 / 143})=37.82191632128952 \\
& y_{159}(i \sqrt{5 / 159})=28.8795746448 \\
& y_{174}(i \sqrt{5 / 174})=25.041594579 \\
& y_{182}(i \sqrt{5 / 182})=23.59338662244782 \\
& y_{222}(i \sqrt{5 / 222})=17.4868329805 \\
& y_{231}(i \sqrt{5 / 231})=16.58028122820744 \\
& y_{238}(i \sqrt{5 / 238})=16.05160446066119 \\
& y_{37}(i \sqrt{7 / 37})=3684.073263147888 \\
& y_{43}(i \sqrt{7 / 43})=2035.247446686725 \\
& y_{53}(i \sqrt{7 / 53})=974.86149890016 \\
& y_{57}(i \sqrt{7 / 57})=749.0991994453120 \\
& y_{58}(i \sqrt{7 / 58})=726.05402077238 \\
& y_{61}(i \sqrt{7 / 61})=610.92037960831 \\
& y_{65}(i \sqrt{7 / 65})=502.6022494543922 \\
& y_{74}(i \sqrt{7 / 74})=351.366679207434 \\
& y_{79}(i \sqrt{7 / 79})=280.680572480 \\
& y_{82}(i \sqrt{7 / 82})=260.001597867 \\
& y_{83}(i \sqrt{7 / 83})=245.455540371 \\
& y_{86}(i \sqrt{7 / 86})=229.550789544 \\
& y_{89}(i \sqrt{7 / 89})=204.133969970 \\
& y_{101}(i \sqrt{7 / 101})=154.17382861 \\
& y_{102}(i \sqrt{7 / 102})=145.39819555 \\
& y_{111}(i \sqrt{7 / 111})=114.058279641 \\
& y_{114}(i \sqrt{7 / 114})=112.1237582645306 \\
& y_{118}(i \sqrt{7 / 118})=104.0227741 \\
& y_{123}(i \sqrt{7 / 123})=94.81858372 \\
& y_{130}(i \sqrt{7 / 130})=88.84241761564202 \\
& y_{131}(i \sqrt{7 / 131})=82.963926585 \\
& y_{138}(i \sqrt{7 / 138})=74.528147461 \\
& y_{141}(i \sqrt{7 / 141})=71.18188653 \\
& y_{142}(i \sqrt{7 / 142})=70.37199791 \\
& y_{143}(i \sqrt{7 / 143})=69.198576423132 \\
& y_{145}(i \sqrt{7 / 145})=63.29462277722419 \\
& y_{155}(i \sqrt{7 / 155})=59.19193845852319 \\
& y_{159}(i \sqrt{7 / 159})=52.6117098
\end{aligned}
$$


$x_{174}(i \sqrt{7 / 174})=16.0822472$

$x_{190}(i \sqrt{7 / 190})=14.92489908685281$

$x_{195}(i \sqrt{7 / 195})=14.24499799839840$

$x_{222}(i \sqrt{7 / 222})=9.76157718$

$x_{210}(i \sqrt{11 / 210})=22.31070843517429$ $y_{174}(i \sqrt{7 / 174})=44.2948363$
$y_{190}(i \sqrt{7 / 190})=41.36554273317740$
$y_{195}(i \sqrt{7 / 195})=32.73499399519519$
$y_{222}(i \sqrt{7 / 222})=28.8422706$
$y_{210}(i \sqrt{11 / 210})=79.24283374069717$

\section{Appendix D. Polynomials $\mathcal{M}(X)$}

Here we list minimal polynomials $\mathcal{M}(X)$ for $x_{N}\left(\tau_{0}\right)$ for various $N$ and $\tau_{0}$. These polynomials are also generating polynomials for the class field $\mathcal{O}$ over $K$.

$N=37, \tau_{0}=-1 / 2+i \sqrt{3 / 4}, d_{K}=-3: \mathcal{M}(X)=X^{12}-52764 X^{11}-1411024 X^{10}+3479640 X^{9}+658332726 X^{8}+$ $12910496448 X^{7}+136570834052 X^{6}+923207817960 X^{5}+4187011679761 X^{4}+12762329218332 X^{3}+25193149293828 X^{2}+$ $29190173495040 X+15106542566400$

$N=37, \tau_{0}=-1 / 2+i \sqrt{11 / 4}, d_{K}=-11: \mathcal{M}(X)=X^{36}-1122593362 X^{35}-1401945830129 X^{34}-1630443592403868 X^{33}$ $394818332142327581 X^{32}-47396404088249827226 X^{31}-3531061340536804334451 X^{30}-182693132290894149962416 X^{29}-$ $7027845157448622682933814 X^{28}-210323680158319826367645252 X^{27}-5055381310292388604486707738 X^{26}-9989105923787907$ $1651188574195515194154267391338 X^{24}-23140513604715395217869150196964 X^{23}-277812411906924904322759656391862 X^{22}-$ $2880218216640399814383292997018128 X^{21}-25947371499935478303885384253120427 X^{20}-20408221237210390143604872895990$ $1406241044001305282108038580118764517 X^{18}-8508875019262604736803096027501387164 X^{17}-452704312887563554984656740$ $211854642343821078015057541591878138626 X^{15}-871512860226600504765229308205790179015 X^{14}-31464327067746155361217$ $9942520401889293530997604912683817565840 X^{12}-27390445561791619601797111885206545912640 X^{11}-65431887419124759251$ $134574133398692023698054049792447650259456 X^{9}-236064004373403262545849445864084773015552 X^{8}-3488254287800613627$ $427078423959931849232206396085337141432064 X^{6}-423552700037176250726227212705272637538304 X^{5}-3295337739512398628$ $191733745504994172351472540097344176128000 X^{3}-77189658956040392174327632913579968036864 X^{2}-18606645307713830072$ 1885917594604942279904891227841465679872

$N=43, \tau_{0}=-1 / 2+i \sqrt{3 / 4}, d_{K}=-3: \mathcal{M}(X)=X^{14}-52762 X^{13}-1516995 X^{12}+137280 X^{11}+663172858 X^{10}+$ $14734681156 X^{9}+177119237218 X^{8}+1402822351192 X^{7}+7813803861149 X^{6}+31333100191686 X^{5}+90450798887057 X^{4}+$ $184052869829048 X^{3}+251169598021672 X^{2}+206687951912640 X+77633240726800$

$N=43, \tau_{0}=-1 / 2+i \sqrt{11 / 4}, d_{K}=-11: \mathcal{M}(X)=X^{44}-1122593352 X^{43}-1413171830568 X^{42}-1644670014220800 X^{41}$ $411218733027217912 X^{40}-51493100829868551648 X^{39}-4043389388141131573904 X^{38}-222738713609123495379616 X^{37}$ $9213907733592642316742660 X^{36}-299490197136075435905766912 X^{35}-7898378380761595464641805456 X^{34}-1730518636399409$ $3207033695859348318985518569816 X^{32}-50979415169812887637661747288032 X^{31}-702849281881287643380049563765904 X^{30}-$ $8479413971853883372695040796246560 X^{29}-90163062203013800470837110521901018 X^{28}-84993851783021736523598782859045$ $7136777093633860395914494866755165728 X^{26}-53583930541180222205673475841379444896 X^{25}-36083411654358223165109609$ $2184477292735364884348978041609335010208 X^{23}-11910093428390107034128323213020829343024 X^{22}-58550031710706969284$ $259698490975957370192626854117542945847876 X^{20}-1039450622716791237048621873811532893390400 X^{19}-3752908284888252$ $12211471696735496257238292177520086409068896 X^{17}-35757321050363555905727110355758424281290728 X^{16}-$ $94026806216653289935886924850265811656931744 X^{15}-221421397134418363703415879161278833928050352 X^{14}$ $465277181889654699036378878644429806016546144 X^{13}-868474950348026503858589555016934388518653023 X^{12}$ $1431790447090511322863978094075513598326135080 X^{11}-2069974810725504231442868603126927741798881208 X^{10}$ $2600606305381242658758479043676933714367854304 X^{9}-2806449250548669287135397078499199902710970768 X^{8}-$ $2562144049752584273639078123114652515135811840 X^{7}-1938674697375202809734937801942175723559028992 X^{6}$ $1181211217008430403849235993411481815184448512 X^{5}-554979000024005060594071490891604307731243264 X^{4}-$ $187135560121902082676979098544212244654082048 X^{3}-39237530384679657458007847661817545023109120 X^{2}-32587027991807$ 232040993129471102886200538371912250658816

$N=53, \tau_{0}=-1 / 2+i \sqrt{3 / 4}, d_{K}=-3: \mathcal{M}(X)=X^{18}-53008 X^{17}-716942 X^{16}+23283672 X^{15}+882220089 X^{14}+$ $14325312516 X^{13}+148365467818 X^{12}+1097984812724 X^{11}+6114596964436 X^{10}+26328032751732 X^{9}+88872838463022 X^{8}+$ $236358868284840 X^{7}+494037439460281 X^{6}+803456810681588 X^{5}+996993227321578 X^{4}+912747897241548 X^{3}+$ $581529351848193 X^{2}+230407643505060 X+42774811832196$

$N=53, \tau_{0}=-1 / 2+i \sqrt{11 / 4}, d_{K}=-11: \mathcal{M}(X)=X^{52}-1122626870 X^{51}-1399903264623 X^{50}-1552179105170360 X^{49}$ $332190800326465746 X^{48}-35188044865881338000 X^{47}-2284747314865301165932 X^{46}-102163031392912707376492 X^{45}$ $3386003246747959941306937 X^{44}-87401517949738576457588602 X^{43}-1819584756335290555271527023 X^{42}-31345703751957735$ $455547836278665945873737269049 X^{40}-5669058399944089266937942634934 X^{39}-61116999889377265677491148525055 X^{38}$ $576038605249689016485428872960400 X^{37}-4780417282990643286763246976215764 X^{36}-3511871827447115323495303034930209$ $229248598892366934990672785077685814 X^{34}-1332567141919878815853832042648410004 X^{33}-6898677215720241827246700332$ 
$31730721790906314397120709344229400378 X^{31}-128817555900167180864753328237062712221 X^{30}-455020237308086048330320$ $1354423871026913417590406140202460203413 X^{28}-3117267211220879132517946486808020394690 X^{27}-371871598501156810610$ $11411617472429937206938414939115865622648 X^{25}+104077329267896809377247838146574080262110 X^{24}+479253734181098266$ $1706428200656691199090058804033445672791920 X^{22}+5143035516040180844001642894856372915110684 X^{21}+135809533948852$ $31940883282516323180985197761880803533436562 X^{19}+67484769932694003431466974792731739829313239 X^{18}+$ $128668656593390994787504269422072859977019892 X^{17}+221841508172317040482831016937890593100416097 X^{16}+$ $345997695261757060968915965028703719417357422 X^{15}+487695134365771011105398680230461356729803763 X^{14}+$ $619948598790222261224986962530470981918044240 X^{13}+708444055427913255416273061456105081042239976 X^{12}+$ $724621629992241011669674991567479917877064304 X^{11}+659673855989189292075928874451281875579356562 X^{10}+$ $530706771528331677487274272551719218184711332 X^{9}+373900687220701232045217625129373076776121949 X^{8}+$ $228045259919346150120364699199469599047165282 X^{7}+118617924684828254598754618406688339455294165 X^{6}+$ $51581881949269909449038630272575559394632164 X^{5}+18243529651803093462049941874711277450059020 X^{4}+504100570381834$ $1020791257867189611539419294463181237377456 X^{2}+134724071323906790807038957503655291346752 X+8696035813026693571$

$N=61, \tau_{0}=-1 / 2+i \sqrt{3 / 4}, d_{K}=-3: \mathcal{M}(X)=X^{20}-53010 X^{19}-611157 X^{18}+24832404 X^{17}+842947259 X^{16}+$ $12817052322 X^{15}+125110694803 X^{14}+874958118348 X^{13}+4617568921174 X^{12}+18924363904920 X^{11}+61230831564170 X^{10}+$ $157794340789824 X^{9}+324899280056407 X^{8}+533511121752402 X^{7}+693809048585299 X^{6}+705189302771412 X^{5}+$ $548131374844211 X^{4}+314539923734070 X^{3}+125543343701925 X^{2}+31116723169500 X+3606183662500$

$N=61, \tau_{0}=-1 / 2+i \sqrt{11 / 4}, d_{K}=-11: \mathcal{M}(X)=X^{62}-1122626864 X^{61}-1406639059310 X^{60}-1560620264745032 X^{59}$ $341478297257569083 X^{58}-37175070278270772348 X^{57}-2495940645213235105930 X^{56}-115937658130199076075492 X^{55}$ $4005010553737000188693618 X^{54}-108027494254559652617583436 X^{53}-2355085445883603677460721986 X^{52}-4256472539484035$ $650160335319059710842027723063 X^{50}-8519045384127268077095329096460 X^{49}-96883318374392364166698175346966 X^{48}-$ $965209828364862761679646041617596 X^{47}-8485640254349349408152062609986792 X^{46}-6620470007841303754538643996813806$ $460278451212667990522614707800682114 X^{44}-2858849459662625223361819052541803984 X^{43}-1587575085195698807910192374$ $78695125537455507805890153231279792844 X^{41}-346356792951842076543658992834544683546 X^{40}-133728817140935664631638$ $4409368594480959988303148390208457435703 X^{38}-11583497244351718624575768296713563417484 X^{37}-18407944242858638825$ $28333929405648859387530141794284277789136 X^{35}+407424399331408628550403181149902061394262 X^{34}+222738066041847999$ $9193676855518121465581735479628904172920892 X^{32}+32111676637347808253890199183298393762762728 X^{31}+98924972954871$ $274185289475630931712024032587003441589453176 X^{29}+691306281743859280829825330857212124109210518 X^{28}+$ $1596179010310072216120526429440407297652001736 X^{27}+3389244228155627958998693331172486602301328687 X^{26}+$ $6635736848166439911829678194237034650440034972 X^{25}+11998811669479112978371600657065746949212238074 X^{24}+$ $20054540931807120789491378858359495062550490084 X^{23}+30989395331676487701862121649003338478826513240 X^{22}+$ $44261135726151426479779157087575754000041100684 X^{21}+58389425834898917494194330543401875640637477462 X^{20}+$ $71066735006601772012301322896891369396812730961 X^{19}+79683555162548517274485809341195786307900220810 X^{18}+$ $82152503808136182730538106423455155909818601525 X^{17}+77700444806753675228753554022188730793461953323 X^{16}+$ $67233038543103778529111457446899028305824760565 X^{15}+53049938632682606562138606647227324295161714094 X^{14}+$ $38024350236722342540039057606418032494144768108 X^{13}+24645783719183729998551144523954652066076268074 X^{12}+$ $14367720408282791668277910320990350615601544144 X^{11}+7485115879822956493598349621309906473990723765 X^{10}+$ $3457714793681510758874330223645238715179240844 X^{9}+1402833154608342630391328979870128492764475674 X^{8}+$ $493921856985749363418543875294997868548456316 X^{7}+148629243359775233884836029354097910043857425 X^{6}+$ $37461147718038204667990311206621525026713580 X^{5}+7692016716017490197207700741287079341035356 X^{4}+1235757244727356$ $145681942572776111671710590984522061290480 X^{2}+11206608633669041029695425643639147486400 X+422049142924440242559$

$N=79, \tau_{0}=-1 / 2+i \sqrt{3 / 4}, d_{K}=-3: \mathcal{M}(X)=X^{26}-53010 X^{25}-611379 X^{24}+24522912 X^{23}+847405230 X^{22}+$ $13242780740 X^{21}+134594586218 X^{20}+994905673888 X^{19}+5646266405155 X^{18}+25384807564306 X^{17}+92213134186043 X^{16}+$ $274168072709632 X^{15}+672795281976410 X^{14}+1369642847561204 X^{13}+2318910888462750 X^{12}+3266176964687536 X^{11}+$ $3820247382711717 X^{10}+3695948399415198 X^{9}+2938978649227465 X^{8}+1903348405653600 X^{7}+991079489282816 X^{6}+$ $407532774035584 X^{5}+128983204090624 X^{4}+30238029404160 X^{3}+4934724403200 X^{2}+499580928000 X+23592960000$ $N=83, \tau_{0}=-1 / 2+i \sqrt{3 / 4}, d_{K}=-3: \mathcal{M}(X)=X^{28}-53008 X^{27}-717380 X^{26}+22345764 X^{25}+885112150 X^{24}+$ $15319971568 X^{23}+174439480674 X^{22}+1471792852904 X^{21}+9745613029961 X^{20}+52385649672260 X^{19}+233677532055764 X^{18}+$ $878211550835988 X^{17}+2810561449737433 X^{16}+7717100199295032 X^{15}+18271762326319298 X^{14}+37419519761923520 X^{13}+$ $66368332999168582 X^{12}+101902743470228324 X^{11}+135156664516641836 X^{10}+154225027855788000 X^{9}+150456792330317857 X^{8}$ $124359800033062464 X^{7}+85988585655379008 X^{6}+48857848578993024 X^{5}+22233690537993216 X^{4}+7797083726684160 X^{3}+$ $1979561949696000 X^{2}+324035002368000 X+25692733440000$

$N=89, \tau_{0}=-1 / 2+i \sqrt{3 / 4}, d_{K}=-3: \mathcal{M}(X)=X^{30}-53008 X^{29}-717390 X^{28}+22875644 X^{27}+890810081 X^{26}+$ $15083764476 X^{25}+166504196688 X^{24}+1348642385256 X^{23}+8485236148392 X^{22}+42876593718112 X^{21}+177808073585660 X^{20}+$ $614145548119416 X^{19}+1785108324177558 X^{18}+4397925557147128 X^{17}+9227558341655868 X^{16}+16533904544598648 X^{15}+$ $25323771801897814 X^{14}+33129069072898080 X^{13}+36923538080431588 X^{12}+34900549732215576 X^{11}+27784004159505864 X^{10}+$ 
$18445942692208576 X^{9}+10072395933953116 X^{8}+4436087610918696 X^{7}+1531749054017457 X^{6}+397048094942616 X^{5}+$ $71812865010978 X^{4}+7815637558764 X^{3}+317458997361 X^{2}-11731422780 X+378613764$

$N=131, \tau_{0}=-1 / 2+i \sqrt{3 / 4}, d_{K}=-3: \mathcal{M}(X)=X^{44}-53256 X^{43}+196840 X^{42}+36072876 X^{41}+803257048 X^{40}+$ $10577231264 X^{39}+100590352674 X^{38}+748632973896 X^{37}+4555739149976 X^{36}+23318268773404 X^{35}+102423385314232 X^{34}+$ $391952935156016 X^{33}+1322270804468655 X^{32}+3969465809330543 X^{31}+10684361372758013 X^{30}+25943442674614481 X^{29}+$

$57112085822866559 X^{28}+114446053134430682 X^{27}+209438932361476177 X^{26}+350930380264189577 X^{25}+539466844518769041 X^{2}$ $761974549729012805 X^{23}+989916214943538520 X^{22}+1183590765442302788 X^{21}+1302668548026528617 X^{20}+13194864614629234$ $1229276257148397099 X^{18}+1052262349806885825 X^{17}+826414832379528174 X^{16}+594347401804391454 X^{15}+39047856947494480$ $233647478396655967 X^{13}+126860003405265027 X^{12}+62217820088579087 X^{11}+27409278283935739 X^{10}+10770710093417380 X^{9}+$ $3742297708553468 X^{8}+1136826379892543 X^{7}+297531293754096 X^{6}+65780863462656 X^{5}+11953182938976 X^{4}+$ $1714591604736 X^{3}+182013951744 X^{2}+12700385280 X+435974400$

Department of Mathematics, The City College of New York, Convent Avenue at 138th Street, New York, Ny 10031 USA, E-MAIL: JJORGENSON@MINDSPRING.COM

Department of Mathematics, University of Sarajevo, Zmaja od Bosne 35, 71000 Sarajevo, Bosnia and Herzegovina, E-MAIL: LEJLAS@PMF.UNSA.BA

Alemannenweg 1, 89537 Giengen, Germany, e-mail: holger.then@Bristol.AC.uk 